\title{
Impulsive disturbances of the geomagnetic field as a cause of induced currents of electric power lines
}

\author{
Vladimir Belakhovsky ${ }^{1}, V$ yacheslav Pilipenko ${ }^{2,3, *}$, Mark Engebretson ${ }^{4}$, Yaroslav Sakharov ${ }^{1}$, \\ and Vasily Selivanov 5 \\ ${ }^{1}$ Polar Geophysical, Apatity, Russia \\ ${ }^{2}$ Geophysical Center, Moscow, Russia \\ ${ }^{3}$ Institute of Physics of the Earth, Moscow, Russia \\ ${ }^{4}$ Augsburg University, Minneapolis, USA \\ ${ }^{5}$ Northern Energetics Research Center, Apatity, Russia
}

Received 8 January 2018 / Accepted 8 April 2019

\begin{abstract}
Geomagnetically induced currents (GICs) represent a significant challenge for society on a stable electricity supply. Space weather activates global electromagnetic and plasma processes in the near-Earth environment, however, the highest risk of GICs is related not directly to those processes with enormous energy yield, but too much weaker, but fast, processes. Here we consider several typical examples of such fast processes and their impact on power transmission lines in the Kola Peninsula and in Karelia: interplanetary shocks; traveling convection vortices; impulses embedded in substorms; and irregular Pi3 pulsations. Geomagnetic field variability is examined using data from the IMAGE (International Monitor for Auroral Geomagnetic Effects) magnetometer array. We have confirmed that during the considered impulsive events the ionospheric currents fluctuate in both the East-West and North-South directions, and they do induce GIC in latitudinally extended electric power line. It is important to reveal the fine structure of fast geomagnetic variations during storms and substorms not only for a practical point of view but also for a fundamental scientific view.
\end{abstract}

Keywords: Geomagnetically indices currents (GICs) / impulsive geomagnetic disturbances / Pi3 pulsations

\section{Introduction}

As advanced technologies are implemented more widely, they have become increasingly subject to sensitive failures due to the impact of space weather such as disturbances of the geomagnetic field and the ionosphere. As technology evolves, man-made technological systems may become even more susceptible to geomagnetic disturbances. One of the most significant factors of space weather for terrestrial technological systems is geomagnetically induced currents (GICs) in conductor systems caused by abrupt changes of the geomagnetic field (e.g., Boteler et al., 1998; Lanzerotti, 2001; Knipp, 2015). GICs associated with great magnetic disturbances were found to be dangerous for various technological systems, causing malfunction of railway equipment (Sakharov et al., 2009a; Eroshenko et al., 2010), deleterious impacts on telephone lines

\footnotetext{
*Corresponding author: space.soliton@gmail.com
}

(Pirjola et al., 2005), and reduction of the lifetime of pipelines (Pulkkinen et al., 2001).

There are numerous examples of the noticeable consequences of space weather events for extended high-voltage power grids. Induced currents cause saturation, rapid growth of harmonics, overheating, and even damage of high-voltage transformers (Zanetti et al., 1994; Erinmez et al., 2002). The most intense currents (over hundred of amperes) have been measured in the neutral lines of transformers at auroral latitudes during magnetic storms and substorms. There is no general rule of how large GIC could be harmful, since there are many types of transformers with different sensitivities to GIC (Girgis et al., 2012). For some power transformers, only a few amperes of quasi-DC current are needed to shift the transformer operation from linear regime to nonlinear regime (Vakhnina et al., 2015). Geomagnetic variations with the time derivative of the geomagnetic field $\mathrm{d} B / \mathrm{d} t>1 \mathrm{nT} \mathrm{s}^{-1}$ were found to be sufficient to induce GIC in Finnish power lines of about several amperes and higher, and variations with $\mathrm{d} B / \mathrm{d} t>40 \mathrm{nT} \mathrm{s}^{-1}$ caused 
problems in operation of the Swedish power lines on July 1982 (Viljanen, 1997). The main phase of a geomagnetic storm or onset of a substorm is considered to be a cause of a high $\mathrm{d} B / \mathrm{d} t$, and, therefore, a main risk factor for power systems at high latitudes. For example, the Hydro Quebec collapse was associated with a storm-related $\mathrm{d} B / \mathrm{d} t \sim 8 \mathrm{nT} \mathrm{s}^{-1}$ (Fiori et al., 2014). However, GIC impacts to power systems have been observed at even smaller rates of $\mathrm{d} B / \mathrm{d} t \sim 2 \mathrm{nT} \mathrm{s}^{-1}$ (Kappenman, 2005).

Ongoing expansion of high-voltage power networks, the growth of linkage between them, the increase of load, and the transition to low-resistive transmission lines with a higher voltage lead to an increased probability of failures during space weather events. The expansion of transmission lines over the past few decades has made the power grid the equivalent of a large antenna that is electromagnetically coupled to the perturbations produced by disturbances of the Earth's magnetosphere. Catastrophic failures are not necessarily required in order to have a detectable economic impact in the wholesale electricity markets. During magnetic storms, the upper limit of the energy transfer is reduced by operators and shortage of energy leads to an increase in the current regional prices. For example, for the storm of July 2000 the prices in the biggest US electrical network jumped almost 4-fold (Forbes \& St. Cyr, 2004). Econometric analysis has shown that a lengthy $10 \%$ reduction in electricity supply to highly industrialized regions due to a probable failure of expensive transformers during severe storm can impact the global economy on the same scale as global financial crisis (Schulte in den Baumen et al., 2014). Although, it is now believed that the most likely consequence of an extreme geomagnetic storm, if not mitigated, is a widespread system voltage collapse instead of permanent damage to a large number of transformers (Pulkkinen et al., 2017). Schrijver et al. (2014) found that insurance claim rates for industrial electrical equipment across the North America rose significantly on the days with elevated geomagnetic activity. Thus, even if power infrastructure hardware is not lost during severe space weather events, GICs in regional power grids can still have broad flow-on economic effects.

The most active manifestations of geomagnetic disturbances and GICs were observed at auroral latitudes, so in the highlatitude countries (USA, Canada, and Fennoscandia) research on the impact of GIC on terrestrial technology systems and possible measures to mitigate the adverse effects was initiated (Pirjola, 2000; Pulkkinen et al., 2005, 2015). Nowadays, even middle and low latitude countries have become concerned about the possible impact of GIC on technological systems (Trivedi et al., 2007; Watari et al., 2009; Marshall et al., 2012; Kelly et al., 2016).

Space weather caused by the interaction of solar ejecta with the near-Earth environment activates global electromagnetic and plasma processes: intensification of 3D magnetosphere ionosphere current systems, energization of ring current and radiation belt particles, bursts of precipitation into the auroral oval, etc. The total amount of power released during a medium magnetic storm is $\sim 1400 \mathrm{GW}$, which is twice as much as the yield of all US power plants, whereas $\sim 70 \%$ of total energy output is dissipated in high-latitude ionosphere (Li et al., 2012). However, the highest risk of GIC may not be related directly to those processes with enormous energy yield, but too much weaker, but fast, processes. Though the power of such processes is many orders of magnitude lower than the power of magnetospheric storms and substorms, the rapidly varying electromagnetic fields of these events can produce a significant GIC.

GIC were previously often modeled as fluctuations of intensity of the East-West auroral electrojet producing geoelectric fields in the longitudinal direction (Boteler et al., 1998). On the basis of these notions, it was commonly supposed that geomagnetic disturbances are most dangerous for technological systems (like power lines and oil/gas pipe lines) extended in the longitudinal direction. However, it was found that fast smallscale ionospheric current structures can provide a significant contribution to rapid geomagnetic field variations, responsible for GIC generation (Viljanen, 1998; Viljanen et al., 2001). Thus, to characterize the geomagnetic field variability, one needs finer characteristics than the widely used time derivative of the $X$-component (North-South) of the geomagnetic field $\mathrm{d} X /$ $\mathrm{d} t$. It is still tempting to find an adequate tool to reveal the temporal-spatial features of geomagnetic field variations most relevant to the GIC generation. For this purpose, Pulkkinen et al. (2006) suggested to apply the structure function analysis and revealed significant change in the dynamics of the magnetic field fluctuations around $80-100 \mathrm{~s}$ where $\mathrm{d} B / \mathrm{d} t$ underwent a transition from correlated to uncorrelated temporal behavior. Also it was found that spatial symmetry of the fluctuations of horizontal magnetic field vector increased during substorms indicating the presence of spatially less ordered ionospheric equivalent currents.

We hope that detailed case studies of selected events with actual GIC recording data will stimulate the geophysical community to find the best data analysis tool for further development of adequate statistical models. Here we consider several examples of fast impulsive processes and their impact on power transmission line:

- sudden commencements (SCs) and sudden impulses (SIs);

- day-side travelling convection vortices (TCVs);

- night-side magnetic impulse events embedded into substorms;

- irregular Pi3 pulsations.

Some types of these impulsive events (e.g., SC/SI) were discussed already in regard to GIC excitation, and they have been added for completeness. However, some types have not been discussed so far as a possible GIC driver (TCVs, impulses embedded in substorms, and Pi3 pulsations), to the best of our knowledge. Moreover, the progress of studies of the GIC mechanisms and drivers is hampered by the fact that almost all information on GIC recordings belongs to commercial companies and is not available for the scientific community for scrutiny analysis. Therefore, any additional observational results on a GIC event is helpful for development of adequate models.

We examine the contribution of geomagnetic disturbances using the International Monitor for Auroral Geomagnetic Effects (IMAGE) array of magnetometers in Fennoscandia during various space weather events to enhancements of GIC recorded by a dedicated recording system in an electric power line in the Kola Peninsula. Detailed examination of possible GIC drivers is important not only from a practical point 
of view, but also has a fundamental scientific interest, revealing the fine structure of fast geomagnetic variations during storms and substorms.

\section{Data and methods}

A system to monitor the impact of GIC on power lines was deployed in 2010 in the Kola Peninsula and Karelia by the Polar Geophysical Institute and the Center of Nordic Energetics (Sakharov et al., 2007) in the frameworks of the European Risk from Geomagnetically Induced Currents (EURISGIC) project (Viljanen, 2011). The system consists of four stations at $330 \mathrm{kV}$ power line (VKH, LKH, KND) and a station RVD at the $110 \mathrm{kV}$ power line (see the map in Fig. 1 and station coordinates in Table 1). The power lines and transformers of low-voltage grids have a higher resistance. GIC in power lines are hard to measure directly, that is why current at a substation is measured in the grounded neutral of the transformer. This current is composed from the currents flowing to the grounding point from all lines connected to the substation. Lines can have a different direction, so GIC in power lines may differ from the current in the neutral. Each station records a quasi-DC current in the dead-grounded neutral of a transformer with 10-s sampling rate. However, this current is not always the same as GIC in a transmission line, because the number of transformers connected to a power transmission line at each moment is beyond our control. The configuration of a power grid may vary from time to time, for example, some lines may be temporarily disconnected. Thus, comparing GIC amplitudes at different grounding points is not meaningful, because the measured currents at sub-station transformers do not correspond directly to GICs in the power lines. Moreover, GIC data are not always available from every station. Preliminary analysis of GIC system data showed that not every strong geomagnetic disturbance necessarily produced intense GIC (Sakharov et al., 2009b). Substantial GIC were mostly recorded under elevated geomagnetic conditions (planetary index $K_{\mathrm{p}}>3$ ). The statistical relationship between the GIC magnitude and $|\mathrm{d} X / \mathrm{d} t|$ peak magnitude demonstrated a tendency of correlation, but the scatter was large.

The variations of the geomagnetic field were measured by the IMAGE three-component magnetometers with 10-sec time resolution (see the map in Fig. 1 and the coordinates in Table 1). The $X$-component corresponds to the North-South geographic direction, $Y$-component corresponds to the East-West direction, and $Z$-component is vertical. Combining nearest magnetometers and GIC stations the following pairs of sites at the same geomagnetic latitudes have been formed: VKH-LOZ/IVA and KND-MEK.

To characterize the geomagnetic field variability in magnitude and direction, the following characteristics have been applied. A geomagnetic disturbance can be presented as a time sequence of snap-shots of the vector field of horizontal magnetic field disturbances $\boldsymbol{\Delta} \boldsymbol{B}$ in latitude-longitude coordinates (e.g., this technique is incorporated in the SuperMAG online system http://supermag.jhuapl.edu). The disturbance is measured as deviation from the reference level $\boldsymbol{B}_{\mathrm{o}}$ before the onset of a disturbance, namely $\Delta \boldsymbol{B}(t)=\boldsymbol{B}(t)-\boldsymbol{B}_{\mathrm{o}}$. However, such an approach would be adequate only for a sufficiently dense $2 \mathrm{D}$

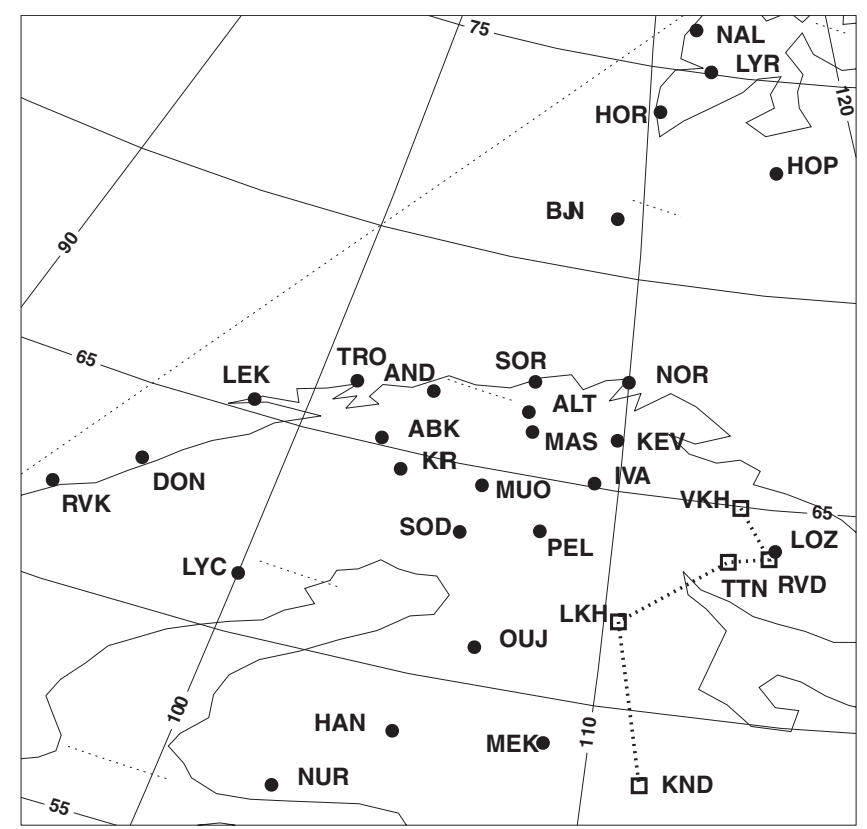

Fig. 1. A map showing the locations of stations for recording GIC in power lines (empty squares) and magnetometers from the IMAGE array (black dots). Dotted line shows the power transmission line. Geomagnetic coordinates are shown by solid lines, and geographic coordinates are denoted by dashed lines.

array of stations. For magnetometers elongated along a meridian it would be more practical to use another approach. For an array of magnetometers oriented along a geomagnetic meridian, the vector diagram method can be applied. This technique presents in a concise form the time evolution of the meridional profile of the horizontal vector of the magnetic disturbance. For that, vectors of geomagnetic disturbances $\Delta \boldsymbol{B}=(\Delta X, \Delta Y)$ for each station are plotted on the same plot as the time sequence of vectors. This technique was used in Friis-Christensen et al. (1988) for the analysis of TCVs. The same diagram can be constructed for the equivalent ionospheric current $\boldsymbol{J}$ and the time derivative of the horizontal magnetic field vector $\mathrm{d} \boldsymbol{B} / \mathrm{d} t=$ $(\mathrm{d} X / \mathrm{d} t, \mathrm{~d} Y / \mathrm{d} t)$. The equivalent current $\boldsymbol{J}$ is related to $\Delta \boldsymbol{B}$ as follows $\Delta \boldsymbol{B}=\left(\mu_{\mathrm{o}} / 2\right)[\boldsymbol{J} \times \boldsymbol{n}]$, where $\boldsymbol{n}$ is the normal to the ground surface, so the vector $\boldsymbol{J}$ is $\pi / 2$ clockwise rotated as compared with $\Delta \boldsymbol{B}$.

These simple relationships are good for a qualitative estimate, but for more precise equivalent current modeling at ionospheric altitudes, the method of spherical elementary current systems can be used (Amm \& Viljanen, 1999; Pulkkinen et al., 2003). The Finnish Meteorological Institute provides the online (http://space.fmi.fi/image/beta/) capability to compute and visualize $2 \mathrm{D}$ ionospheric equivalent current vectors from the IMAGE magnetometers using this method. The method is based on the fact that the horizontal ionospheric currents can be divided into divergence-free and curl-free components. The curl-free horizontal currents close the field-aligned currents linking the upper atmosphere with magnetospheric processes, and this combined system does not produce any magnetic field below the ionosphere. The technique determines from groundbased magnetometer data the divergence-free component of 
Table 1. Magnetic and GIC stations.

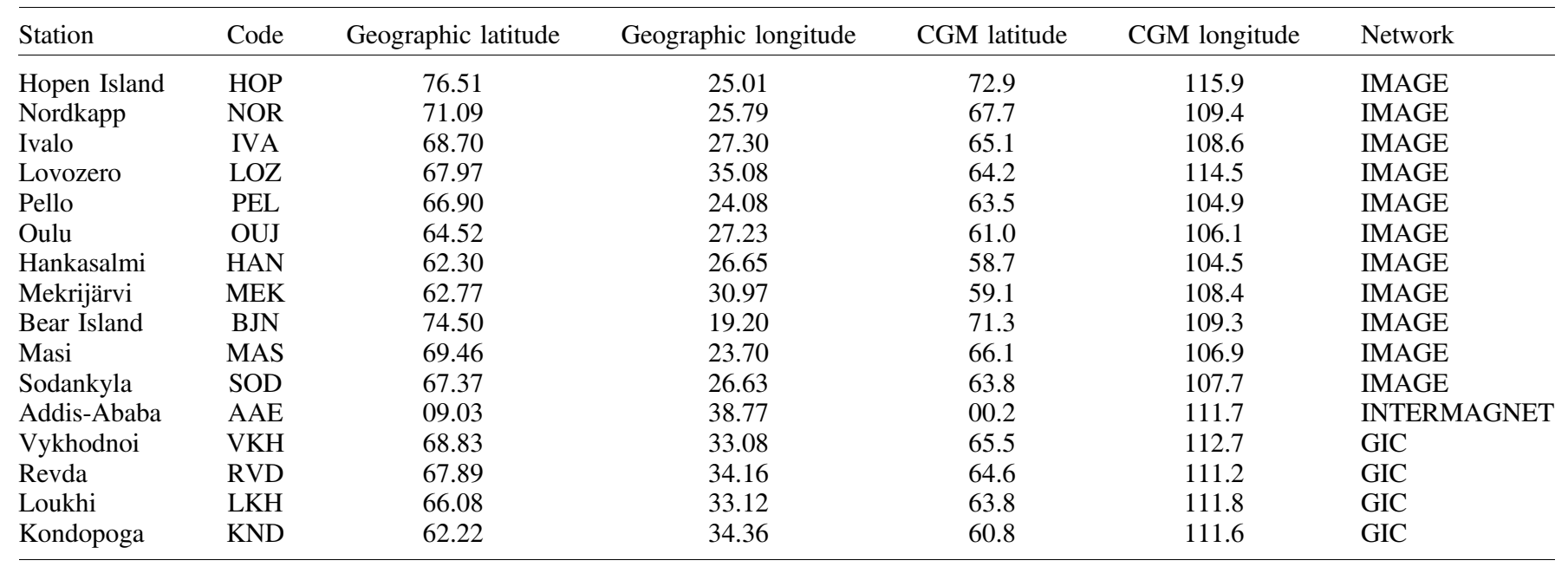

the equivalent ionospheric currents (which roughly describes the distribution of ionospheric Hall currents).

\section{GIC stimulation by SC/SI}

The impact of an interplanetary shock onto the magnetosphere is observed by ground magnetometers as a storm sudden commencement (SC) or sudden impulse (SI) prior to the storm main phase (Araki, 1994). This impact is often used as a convenient probing signal for the experimental study of the near-Earth space. During a relatively short time period (<10 min), a significant energy and momentum are pumped into the magnetosphere. Despite the seeming simplicity of such an impact, the complexity of impulsive and quasi-periodic phenomena stimulated by an interplanetary shock in the time scales from few seconds to tens of minutes turns out to be surprisingly large (Belakhovsky et al., 2017). The magnitudes of SC that mark the onset of the storm are not correlated with the size of its main phase. Furthermore, the intensity of the auroral zone current, characterized by the auroral electrojet (AE) index, is not necessarily correlated with the depth of the main phase minimum. Since these three current systems show much independence, even though causally linked, extremely strong interplanetary shocks may precede a very moderate storm main phase.

The interplanetary shock impact deserves a special consideration as a driver of GICs. Although the geomagnetic disturbance $\Delta \boldsymbol{B}$ associated with $\mathrm{SC} / \mathrm{SIs}$ is small compared to $\Delta \boldsymbol{B}$ during storm/substorm, the relevant time derivative $\mathrm{d} \boldsymbol{B} / \mathrm{d} t$ can still be large enough to induce dangerous GICs in power systems (Kappenmann, 2003). Disturbances with $\mathrm{d} B / \mathrm{d} t>2 \mathrm{nT} \mathrm{s}^{-1}$ can occur during the arrival of an interplanetary shock in the auroral region (Fiori et al., 2014), and even at equatorial stations (Carter et al., 2015). As an example, the failure of transformer of the New Zealand power system reported by Béland \& Small (2004) coincided with a SC on November 6, 2001. Emergency shutdown at the power substation Olenegorsk in Northern Russia took place during SC on October 29, 2003 (Sakharov et al., 2007). During an SC in August 1972, the electric power system in the northern part of the US experienced major disturbances and a complete outage of the continental telecommunication cable occurred (Anderson et al., 1974). During this SC, the time rate of magnetic field change in the region where communication disruption occurred was $\sim 12 \mathrm{nT} \mathrm{s}^{-1}$. Some power system failures at low latitude have been associated with the occurrence of the SC on March 17, 2015 (Zhang et al., 2015).

Here we present an example of a burst of GIC in the power lines recorded by the station VKH during the SI of 2014, December 23 (Fig. 2). The responsible interplanetary shock was recorded by the interplanetary monitor ACE (Advanced Composition Explorer) as a sudden jump of the solar wind velocity from $\sim 330$ to $>500 \mathrm{~km} \mathrm{~s}^{-1}$, plasma density from $\sim 8$ to $\sim 18 \mathrm{~cm}^{-3}$, and vertical component $B_{\mathrm{z}}$ of the interplanetary magnetic field (IMF) from $\sim 10$ to $\sim 30 \mathrm{nT}$ on the background of northward IMF. Figure 2 gives a comparison of the geomagnetic field and GIC response to SI (left panel) and subsequent substorm (right panel).

An isolated magnetic pulse is recorded by all stations along the geomagnetic profile from auroral latitudes to the geomagnetic equator (station AAE) (the middle panel of the left-side plot of Fig. 2). The global character of SC discriminates this type of magnetospheric response from localized auroral disturbances, such as TCV. At high-latitude stations (BJN, NOR) the magnetic field compression is preceded by a preliminary negative impulse.

During the SI, a burst of GIC is recorded at VKH with peakto-peak amplitude $\sim 30 \mathrm{~A}$. Variations of the GIC are similar to variations of $-\mathrm{d} X / \mathrm{d} t$ at the magnetic station IVA located at the same geomagnetic latitude. During the SI, the $|\mathrm{d} X / \mathrm{d} t|$ peak-to-peak level reaches $\sim 2.5 \mathrm{nT} \mathrm{s}^{-1}$.

The GIC peak-to-peak intensity caused by the SC ( 30 A) is about two times higher than the GIC during the subsequent substorm intensifications at $\sim 20: 40$ UT $(\sim 15 \mathrm{~A})$, though at IVA the SC amplitude ( 100 nT) is less than the amplitude of the substorm-associated magnetic bay (up to $400 \mathrm{nT}$ ). Thus, daytime SC/SI may have an effect on terrestrial systems comparable or even greater than those nighttime effects associated with substorms. 

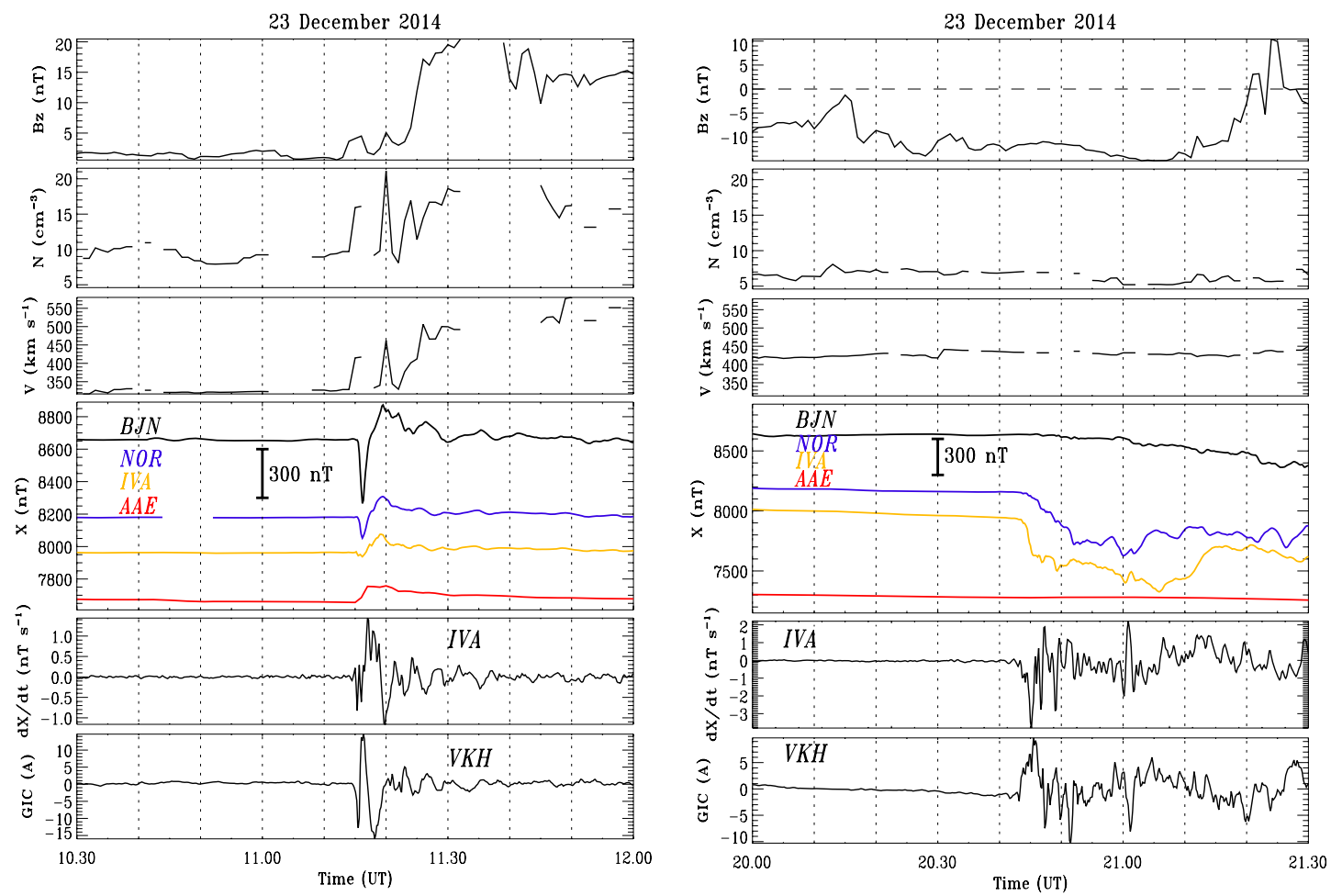

Fig. 2. The interplanetary shock and substorm impacts on geomagnetic fields and electrical power lines during 23 December 2014 : SC (left panel) and substorm onset (right panel). The panels from top to the bottom are the solar wind density $N$ and velocity $V$ (1-min data from the OMNI database); magnetic records ( $X$-component) from the geomagnetic stations BJN, NOR, IVA (10-s data), and AAE (1-s data); time derivative of magnetic field $\mathrm{d} X / \mathrm{d} t$ [nT s${ }^{-1}$ ] at the station IVA; and GIC [Amperes] at the station VKH (10-s data).

\section{Day-side traveling convection vortices and GIC}

The interaction between the solar wind and the magnetosphere serves as a source of diverse types of nonstationary processes and perturbations of different spatial and temporal scales. As a result, intense disturbances can be observed not only in the period of high magnetospheric activity (magnetic storms and substorms), but also in a quiet geomagnetic situation. Such daytime high-latitude impulsive perturbations are now understood to be TCVs, which are a response to a local impact on the magnetosphere (Friis-Christensen et al., 1988; Engebretson et al., 2013). The terrestrial manifestation of a TCV is an isolated magnetic impulse event (MIE) - sporadic perturbation of the geomagnetic field with a duration of $\sim 5-10$ min and with amplitude of 100 nT (Lanzerotti et al., 1990; Vorobjev et al., 1999). The physical mechanism of excitation of a TCV and its interaction with the ionosphere is not uniquely determined. In the picture of equivalent ionospheric currents, a TCV manifests itself as a double vortex of Hall currents with characteristic dimensions up to $\sim 10^{3} \mathrm{~km}$, driven by a pair of upward and downward field-aligned currents between the ionosphere and the magnetosphere (McHenry \& Clauer, 1987).

Here we present an example of a TCV on 26 January 2012, identified in magnetometer data from the IMAGE array (Fig. 3). An isolated bi-polar impulse is recorded at 07:10 UT on a quiet geomagnetic background. The largest peakto-peak amplitudes, $\sim 400 \mathrm{nT}$ in $X$-component and $\sim 300 \mathrm{nT}$ in
$Z$ component, are observed at geomagnetic latitude $\sim 73^{\circ}$ (HOP). At station IVA, located at the same latitude $\sim 65^{\circ}$ as GIC recording station $\mathrm{VKH}$, the peak-to-peak amplitudes are much weaker, $\sim 40 \mathrm{nT}$ in $X$-component and $\sim 80 \mathrm{nT}$ in Z-component. Correspondingly, the time rate of geomagnetic field variations $\mathrm{d} X / \mathrm{d} t \sim 6 \mathrm{nT} \mathrm{s}^{-1}$ at $\sim 73^{\circ}$ latitude (HOP) is several times larger than those at latitude $\sim 65^{\circ}$ (IVA) $\sim 1 \mathrm{nT} \mathrm{s}^{-1}$. This event produces significant burst of GIC with peak-to-peak amplitude $\sim 5 \mathrm{~A}$.

The 2D spatial structure of ionospheric equivalent current vectors corresponding to the recorded TCV is reconstructed from the IMAGE magnetograms using the method of the spherical elementary current systems. The baseline during the pre-impulse interval has been subtracted. The structure (Fig. 4) shows an intense localized clockwise vortex of ionospheric currents centered slightly off-shore Svalbard. The ionospheric current density reaches $\sim 500 \mathrm{~A} \mathrm{~km}^{-1}$. At Fennoscandia mainland stations this vortex is hardly noticeable, nonetheless, it causes a noticeable burst of GIC. The same vortex, but shifted to lower latitude, would produce at least an order of magnitude larger GIC.

\section{Magnetic impulse events during the substorm of 17 March 2013}

Solitary pulses with large amplitudes can be observed in the night sector at auroral latitudes (Ngwira et al., 2015). These 


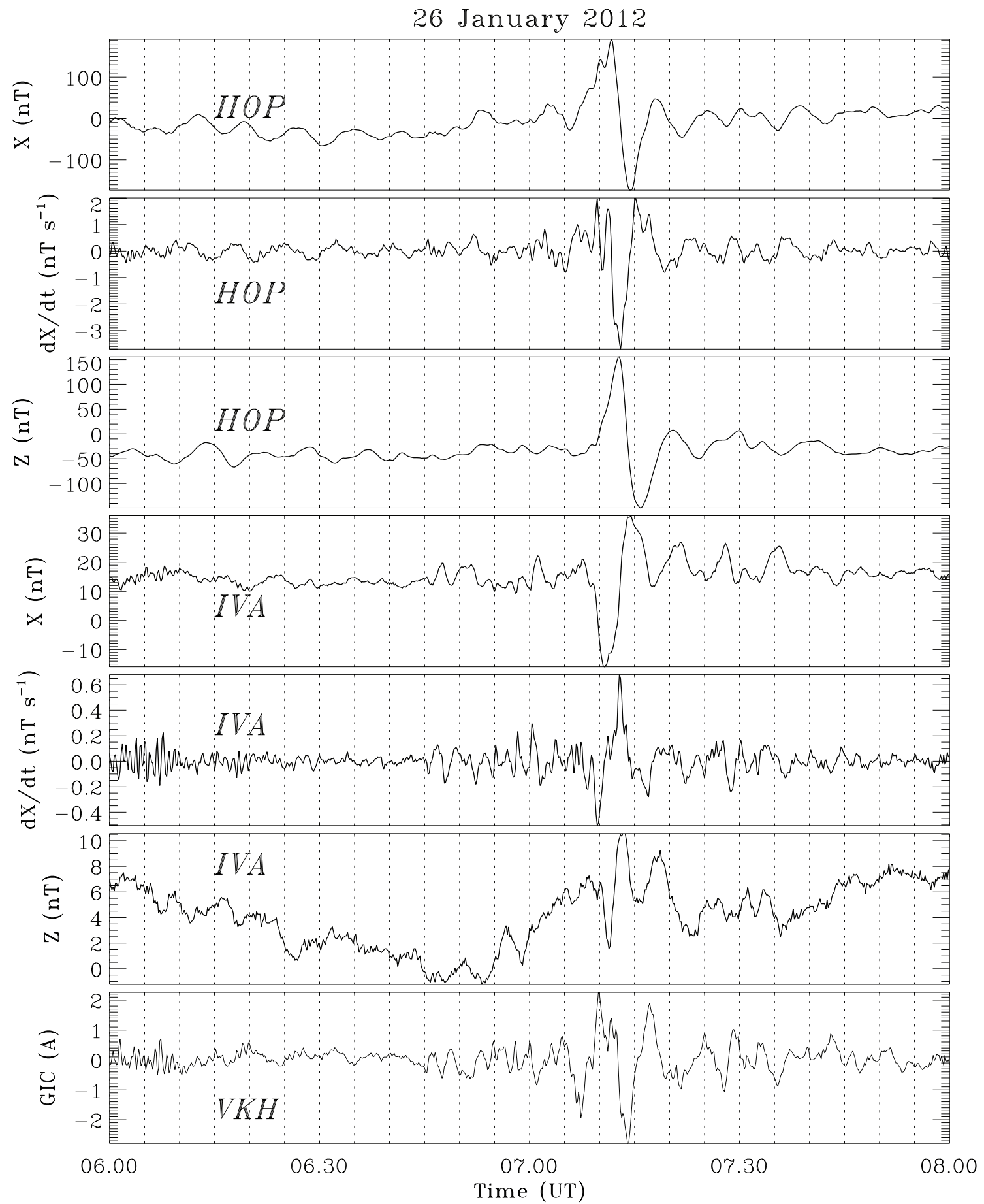

Fig. 3. The $X$ - and Z-components of geomagnetic field at HOP and IVA, the rate of geomagnetic field variations $\mathrm{d} X / \mathrm{d} t$ [nT s ${ }^{-1}$ ] at HOP and IVA (10-s data), and GIC [A] at VKH (10-s data) associated with the TCV between 06 UT and 08 UT on 26 January 2012.

nighttime magnetic pulses are not directly associated with transient solar wind pressure increases, and their origin is unclear. Many night sector magnetic pulses occurred in association with substorm-related magnetic bay, whereas some appeared to be very isolated. It is unknown whether these pulses are part of substorm dynamics, or they are of other origin and just superposed on the substorm process.

Here we present an example of such impulses during the storm on 17 March 2013. During this storm IMF Bz turned southward for a long time providing a long-duration energy 


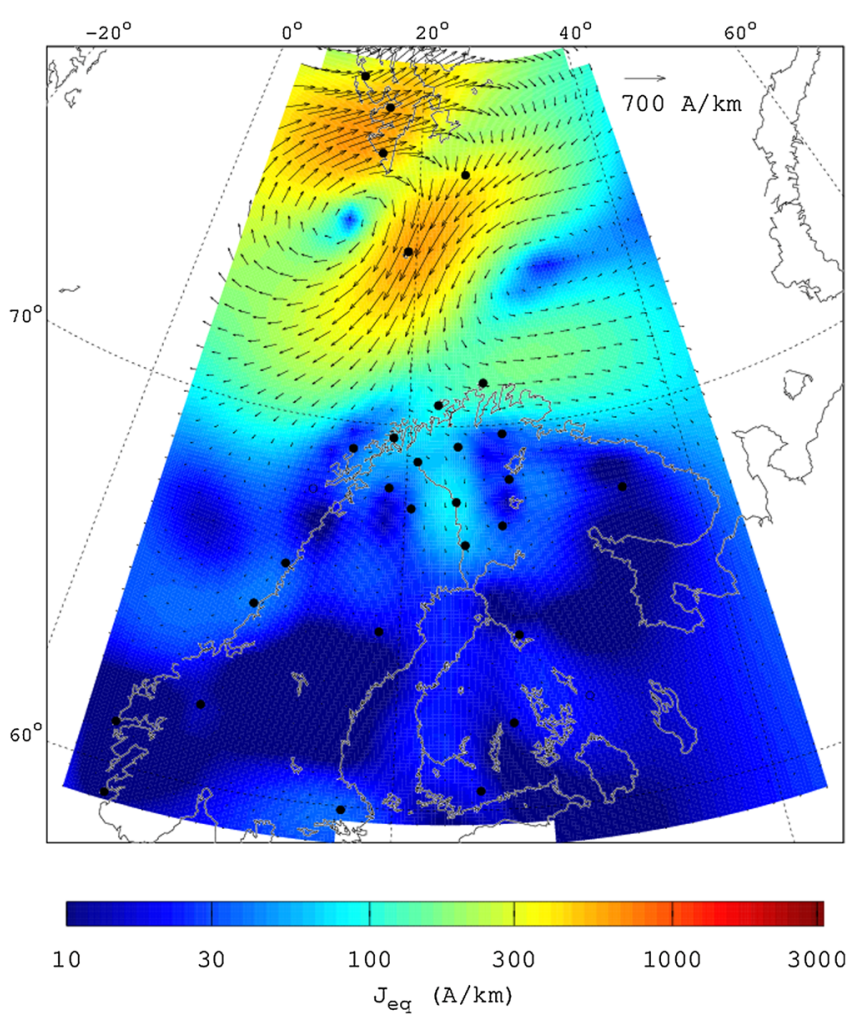

Fig. 4. The $2 \mathrm{D}$ spatial structure of $\mathrm{TCV}$-associated ionospheric equivalent current vectors at 07:13 UT on 26 January 2012, reconstructed from the IMAGE magnetometers using the method of spherical elementary current systems. Color coding indicates the linear density of equivalent ionospheric current $\left[\mathrm{A} \mathrm{km}^{-1}\right]$.

supply into the magnetosphere, which caused a series of substorms. The symmetric (SYM) disturbance index for H component (SYM-H) gradually dropped to $-100 \mathrm{nT}$ and remained on this level. The AE index showed several activations at auroral latitudes and reached up to $\sim 2500 \mathrm{nT}$ at $\sim 17: 00 \mathrm{UT}$.

Geomagnetic field variations at the IMAGE stations during this storm (Fig. 5) in the $X$ component were more intense than in the $Y$ component, that is $|\Delta X|>|\Delta Y|$. On the background of substorm activity, several intense pulses were evident, at $\sim 16: 00, \sim 18: 00$, and $\sim 18: 30$ UT. The impulse at $\sim 16: 00$ UT was the most intense, $\sim 800 \mathrm{nT}$, maximizing at geomagnetic latitude $\sim 68^{\circ}$ (NOR). The impulse was evident not only in the $X$ component, but in the vertical $Z$ component, $\sim 700 \mathrm{nT}$, as well. The impulses during 1800-1830 UT, $\sim 500 \mathrm{nT}$, maximized at lower latitude $\sim 59^{\circ}$ (MEK). They were also evident in both $X$ and $Z$ components. A significant $Z$-component indicates that a primary source of these disturbances is spatially localized structure.

The method of 2D equivalent currents reveals in an evident way that these vortex-like intensifications took place at different locations (Fig. 6). During the impulse at 16:05 UT, the ionospheric current system formed a large vortex with epicenter at latitude $>70^{\circ}$. During the pulses at 18:04 UT and 18:28 UT the intensifications were weaker, but they were shifted to lower latitude $\sim 62^{\circ}$, much closer to the GIC recording station.
During these pulses, the GIC stations recorded significant bursts of GIC intensity (Fig. 7). At higher latitude (VKH), the GIC peak-to-peak variations were as high as $\sim 70 \mathrm{~A}$ during the pulse at $\sim 16: 00$ UT. Two subsequent pulses, at $\sim 18: 00$ UT and 18:30 UT, induced the largest GICs at lower latitude (KND), up to $\sim 22$ A.

The impulse at 16:00 UT marked the onset of AE index growth (Fig. 8a). This activation corresponded to burst of $|\mathrm{d} \boldsymbol{B} / \mathrm{d} t|$ at $\sim 64^{\circ}$ (LOZ) up to $\sim 5 \mathrm{nT} \mathrm{s}^{-1}$ in $X$ component and $\sim 3.7 \mathrm{nT} \mathrm{s}^{-1}$ in $Y$ component, and the GIC magnitude at the near-by station $\mathrm{VKH}$ of $>40 \mathrm{~A}$. Subsequent pulses after $\sim$ 18:00 UT caused less intense GIC response at VKH, because the "epicenter" of burst activity had shifted to lower latitudes. Indeed, at $\sim 59^{\circ}$ (MEK) the impulses at $\sim 18: 00$ UT and $\sim 18: 30$ UT were more evident than the first impulse (Fig. $8 \mathrm{~b}$ ). These pulses corresponded to bursts of $|\mathrm{d} \boldsymbol{B} / \mathrm{d} t|$ (up to $\sim 10 \mathrm{nT} \mathrm{s}^{-1}$ in the $X$ component and $\sim 8.3 \mathrm{nT} \mathrm{s}^{-1}$ in the $Y$ component) and GIC intensity at nearby station $\mathrm{KND}>12 \mathrm{~A}$.

There are no one-to-one correspondences between the substorm intensity characterized by the $\mathrm{AE}$ index, magnetic impulse intensity, and GIC magnitude. For example, intense magnetic pulses and GIC bursts occurred between 18:00 UT and 19:00 UT, when the AE index was somewhat decreased. The local IMAGE electrojet (IE) index (http://space.fmi.fi/ image/www/) might be a better indicator of local susbtorm activity than the global auroral electrojet (AE) or the SuperMAG auroral electrojet (SME) indices (http://supermag.jhuapl. edu/indices) (Newell \& Gjerloev, 2011). Nonetheless, there is still no linear relation between the substorm intensity as measured by any of those indices and the level of geomagnetic variability and GIC. Thus, AE/IE indices are not very reliable measures of related GIC intensity.

Comparison of the magnitude of magnetic disturbances $|\Delta X|$ and $|\Delta Y|$ with the amplitudes of time derivatives $|\mathrm{d} X / \mathrm{d} t|$ and $|\mathrm{d} Y / \mathrm{d} t|$ (Fig. 8a, b) shows that though the magnetic disturbance is much larger in the $X$-component than in the $Y$-component, $|\Delta X|>|\Delta Y|$, their time derivatives $|\mathrm{d} X / \mathrm{d} t|$ and $|\mathrm{d} Y / \mathrm{d} t|$ are comparable. Therefore, small $|\Delta Y|$ does not mean small $|\mathrm{d} Y / \mathrm{d} t|$, and variations of both components provide a similar contribution to the increase of $|\mathrm{d} \boldsymbol{B} / \mathrm{d} t|$.

The array of available mid-latitude IMAGE magnetometers is mostly oriented in the latitudinal direction, along a geomagnetic meridian. The vector diagram technique (Fig. 9) shows that during the impulsive disturbance at $~ 16: 00 \mathrm{UT}$, the equivalent ionospheric currents at the high-latitude end of the profile suddenly change their direction from predominantly East-West (E-W) to North-South (N-S). This transition looks like an occurrence of a vortex-like current system. Although the large-scale structure of the ionospheric currents is determined by the E-W electrojet, at a smaller regional level, the currents experienced strong variations in direction. A series of similar vortex-like disturbances of the ionospheric current occurred at the low-latitude end of the profile between 18:00 UT and 19:00 UT.

The direction of telluric electric field $\boldsymbol{E}$ corresponds to the direction of the vector $\mathrm{d} \boldsymbol{B} / \mathrm{d} t$, rotated by $90^{\circ}$ anticlockwise (strictly speaking, this is valid for a plane incidence field and horizontally homogeneous conductivity of the Earth's crust) (Viljanen et al., 2015). The vector diagram of local $\mathrm{d} \boldsymbol{B} / \mathrm{d} t$ shows that the induced geoelectric fields during the magnetic impulses are to be randomly oriented in all directions (Fig. 10). 

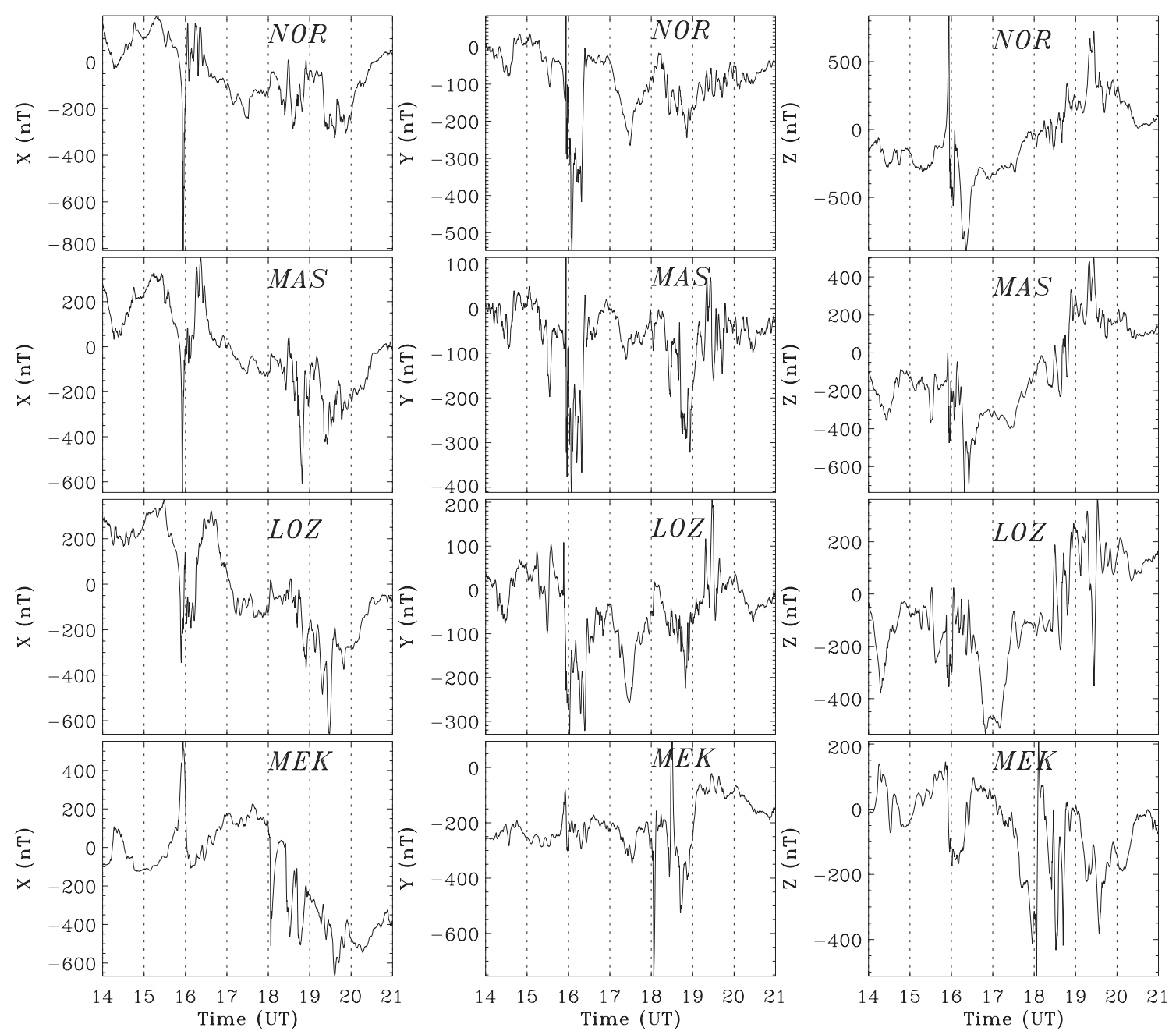

Fig. 5. Geomagnetic field variations at the latitudinal array of stations NOR, MAS, LOZ, and MEK (10-s data) from the IMAGE array during the magnetic storm on March 17, 2013: $X$-, $Y$-, and Z-components.
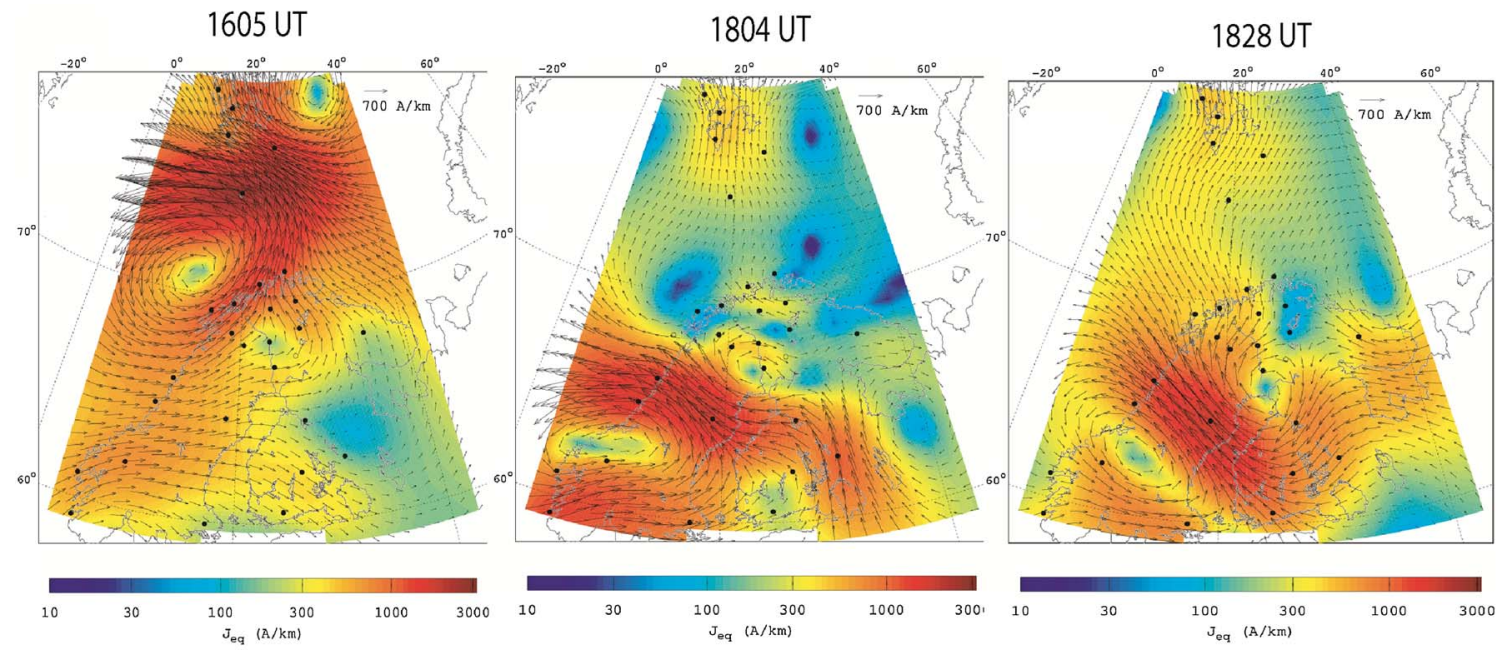

Fig. 6. The equivalent ionospheric current systems calculated with the method of 2D equivalent currents from the IMAGE magnetometer array during three impulses imposed on the substorm evolution at 16:05 UT, 18:04 UT, and 18:28 UT on 17 March 2013. Color coding indicates the density of height-integrated equivalent ionospheric current. 


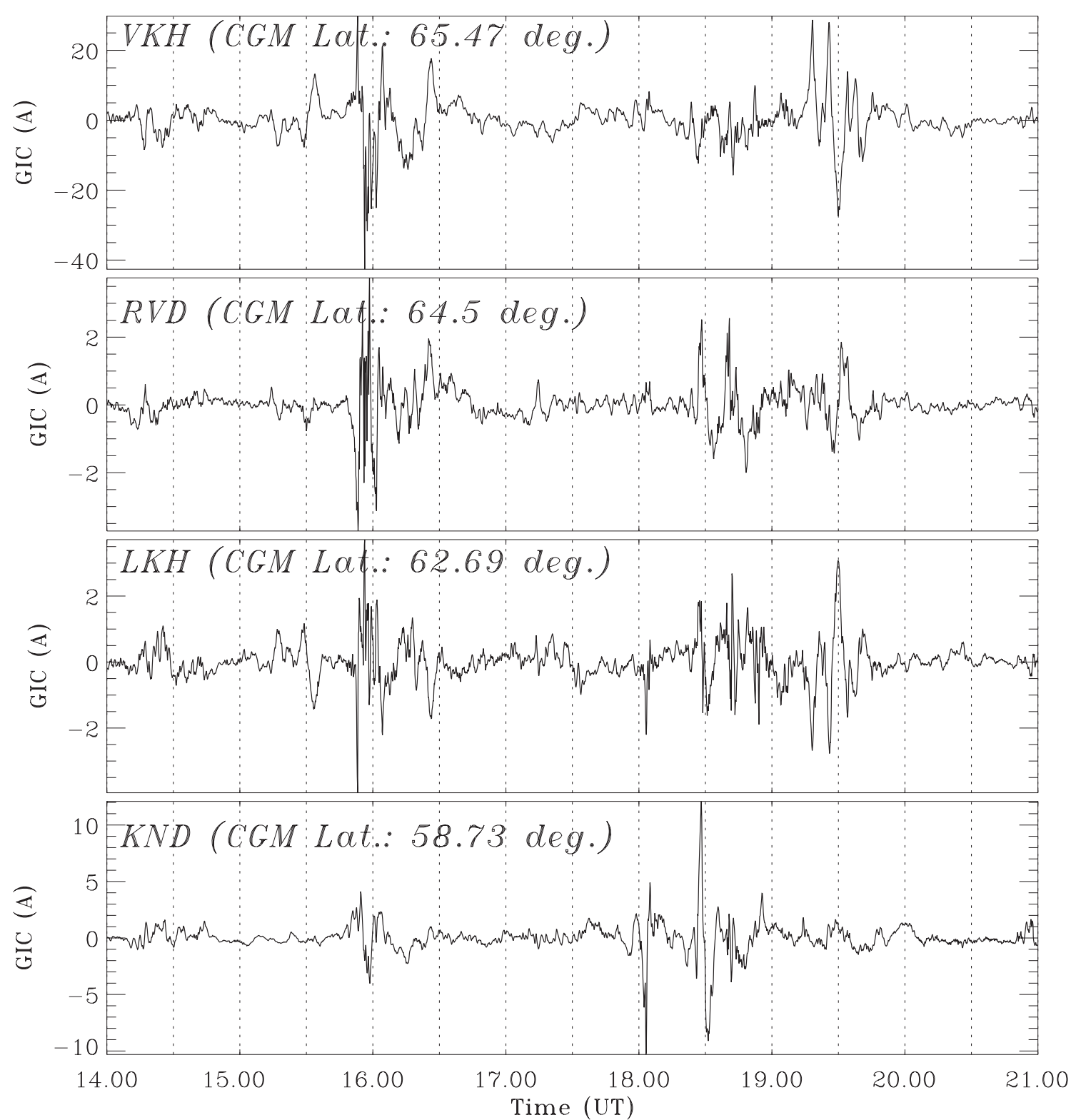

Fig. 7. GIC [A] recorded at stations VHD, RVD, LKH, and KND (10-s data) during the March 17, 2013 magnetic storm. Corrected geomagnetic latitudes are shown near the station codes.

\section{GIC event induced by Pi3 pulsations on 28-29 June 2013}

Pi3 pulsations are in fact the fine structure of a substorm (Saito, 1978). These irregular variations with quasi-period $400-600 \mathrm{~s}(1-3 \mathrm{mHz})$ and amplitudes from several tens of nT to a hundred $\mathrm{nT}$ are localized in latitude in the auroral zone (Nagano et al., 1981). These magnetic pulsations are commonly accompanied by auroral intensity and riometer fluctuations in a similar period range (Kleimenova et al., 2002). Their periodicity is longer than typical Pc5 periods, so they cannot be interpreted as field line eigenoscillations. Their physical mechanism has not been identified firmly yet.
As an example, we consider the magnetic storm on 27-29 June, 2013 that was initiated by an interplanetary shock arrival at $~ 15: 00$ UT on 27 June. The IMF Bz fluctuated around 0, but after 08:00 UT on 28 June, IMF Bz gradually turned southward $(<0)$ and remained steady at about $-10 \mathrm{nT}$ until 12:00 UT on 29 June. The persistent southward IMF drove the magnetosphere into a magnetic storm, during which geomagnetic indices reached maximal values of $|D s t| \sim 120 \mathrm{nT}$ and $A E \sim 1000 \mathrm{nT}$.

This period coincided with a period of maximum of magnetic bay magnitude at the IMAGE magnetic stations (Fig. 11). During the period of maximal magnetic disturbance, intense Pi3 pulsations were superposed on the magnetic bay. These pulsations are not quasi-sinusoidal waves like typical 

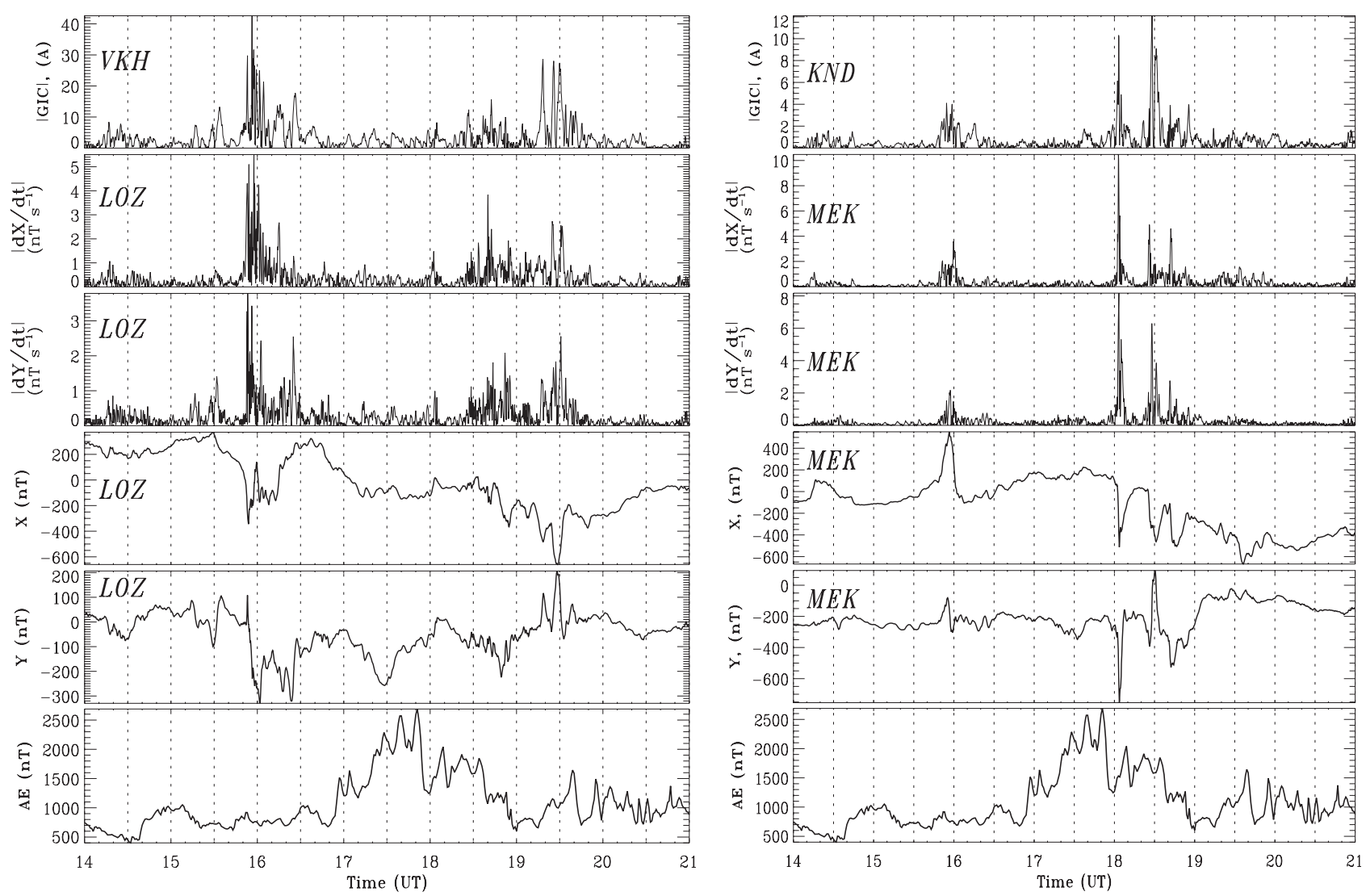

Fig. 8. (a) Comparison of GIC amplitudes [A], magnitudes of derivatives $|\mathrm{dX} / \mathrm{d} t|$ and $|\mathrm{d} Y / \mathrm{d} t|\left[\mathrm{nT} \mathrm{s}{ }^{-1}\right]$, and geomagnetic variations $(X-$ and $Y$-components) [nT] from nearby stations VKH and LOZ during 14-21 UT on 17 March 2013. The bottom panel shows variations of the 1-min $A E$ index. (b) The same format for the KND-MEK pair.

Pc5 pulsations; they are rather quasi-periodic sequences of magnetic impulses. The time scale of these oscillations varies from $\sim 20 \mathrm{~min}$ at lower latitudes up to $\sim 10 \mathrm{~min}$ at higher latitudes. During the maximal geomagnetic disturbance, a burst of magnetospheric energetic electron precipitation occurs, as evident from intense irregular variations of riometer absorption (up to $\sim 50 \%$ ) at SOD (Fig. 11).

During this substorm extremely high values of GIC were recorded (up to $\sim 120$ A per node) at station VKH, from $\sim 01: 00$ to $\sim 03: 00$ UT on 29 June 2013 (Fig. 12).

During the magnetic storm the magnetic disturbance gradually increased and then slowly decayed, and was mainly oriented in the $X$-direction. However, during the maximal disturbance magnetic variations became more chaotic. Comparison of the magnitude of magnetic disturbances $\Delta X$ and $\Delta Y$ with amplitudes of time derivatives $|\mathrm{d} X / \mathrm{d} t|,|\mathrm{d} Y / \mathrm{d} t|$ (Fig. 13) shows that though the magnetic disturbance was much larger in the $X$-component than in the $Y$-component, $|\Delta X|>|\Delta Y|$, the time derivative $|\mathrm{d} Y / \mathrm{d} t| \geq 10 \mathrm{nT} \mathrm{s}^{-1}$ was larger than the time derivative $|\mathrm{d} X / \mathrm{d} t| \geq 8 \mathrm{nT} \mathrm{s} \mathrm{s}^{-1}$. Therefore, variations of both horizontal components provided a similar contribution to the increase of $|\mathrm{d} \boldsymbol{B} / \mathrm{d} t|$.

The vector diagrams of ionospheric current variations for the period between 23:00 UT on 28 June and 06:00 UT on 29 June (Fig. 14) with time cadence 1 min show that the Pi3 pulsations are a sequence of localized vortex-like structures.

\section{Discussion}

Serious economic consequences for the global electricity market may happen even when catastrophic disturbances caused by space weather do not occur. Even if during the magnetic storms there is no loss of technological equipment, GICs in regional power networks have a significant impact on the stability of the economy (Forbes \& St. Cyr, 2008). These and many other examples dictate the need for a deeper study of the impact of space weather on world infrastructure and prediction of the space weather sudden changes.

The existing space monitors at L1 Lagrangian point about 1.5 million kilometers from Earth towards Sun make it possible to detect in real time the arrival of interplanetary shocks with time lag $\sim 1 \mathrm{~h}$. Thus, the risk of SC-associated GIC bursts can be predicted reliably. Though the occurrence of TCV caused by foreshock and magnetosheath transients can hardly be predicted, the associated impulse is not very significant for GIC. Although promising results on forecasting of substorm activity in a real time were achieved using data from interplanetary monitors (Luo et al., 2013), the problem of GIC forecasting is not reduced to the problem of substorm forecasting. The presented events have demonstrated that there is no one-to-one correspondence between the substorm intensity, geomagnetic field variability, and GIC magnitude. The standard global geomagnetic activity measures (such as $A E, K_{\mathrm{p}}, D s t$ indices) 


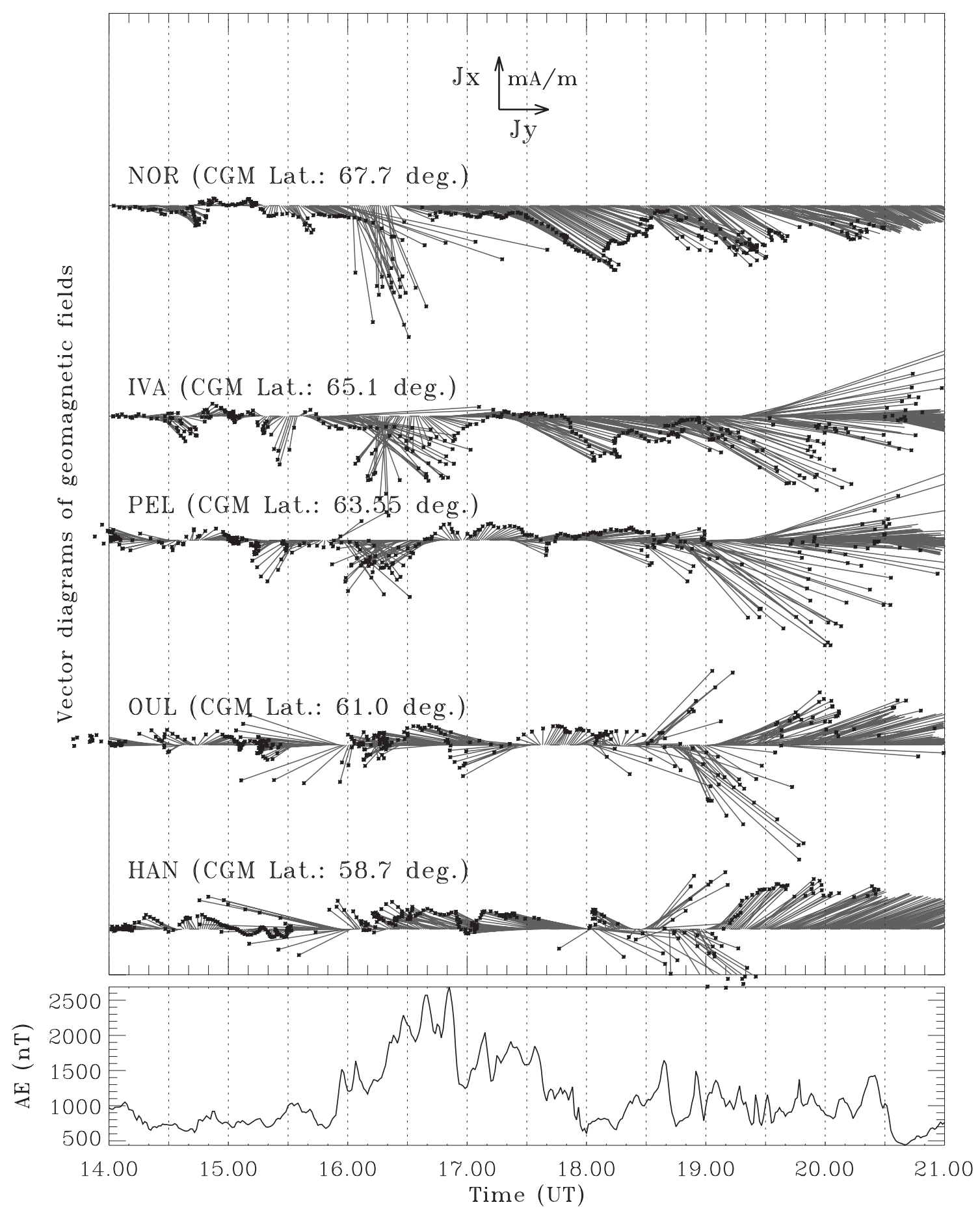

Fig. 9. Vector diagrams of the equivalent currents $\boldsymbol{J}$ along the meridional profile between 14 UT and 21 UT on 17 March 2013 (time step $1 \mathrm{~min})$. The bottom panel shows the 1-min $A E$ index variations.

may fail to reveal significant local GIC events. Therefore, the prediction of time derivatives of the geomagnetic field demands more detailed analysis (Weigel et al., 2003; Juusola et al., 2015; Wintoft et al., 2015). Detailed features of substorm current systems comprising rapid sporadic localized field-aligned currents must be included in the methods of magnetic disturbance description necessary for prediction of GIC. An adequate theory of sporadic magnetic pulses and Pi3 pulsations during substorms has not been advanced enough.

The most intense GIC generation occurs at auroral latitudes, where the most intense disturbances are produced by more localized and dynamic substorm processes. Indeed, at the Kola power line system GIC bursts were observed mainly in nighttime hours during auroral substorms (Sakharov et al., 2009b). 


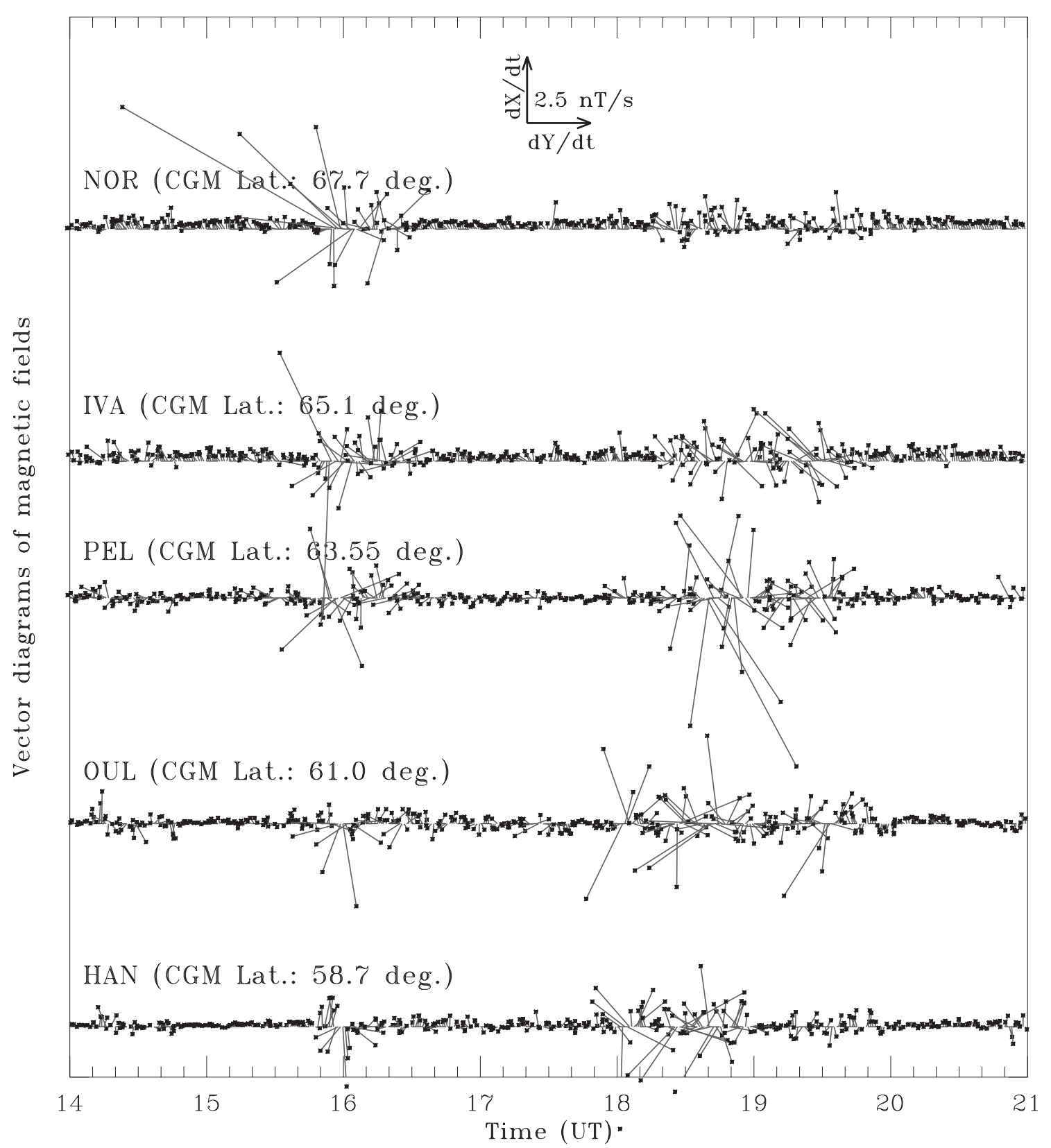

Fig. 10. Vector diagram of the $\mathrm{d} \boldsymbol{B} / \mathrm{d} t$ variations along the meridional profile between $14 \mathrm{UT}$ and $21 \mathrm{UT}$ on 17 March 2013 (time step 1 min).

Our study has provided additional evidence that GIC cannot be interpreted simply as a result of fluctuations of the extended auroral westward electrojet (Viljanen, 1997; Viljanen et al., 2001). The vector technique used here has demonstrated a much larger variability of $\mathrm{d} \boldsymbol{B} / \mathrm{d} t$ in magnitude and direction as compared with just magnetic variations $\Delta \boldsymbol{B}$. Belakhovsky et al. (2018) applied to geomagnetic data the parameter $R B$, which shows how vector fields experience fluctuations in magnitude or in direction. This parameter does not depend on magnitude of a disturbance. A vector field experiences chaotic variations in all directions for $R B \rightarrow 1$ and a field varies in magnitude only for $R B \rightarrow 0$. They found that during magnetic storms $R B \sim 0.7-0.9$ which means that $B$-field experienced chaotic variations not only in magnitude, but in all directions. Thus, such geomagnetic variations cannot be attributed to variations of the East-West auroral electrojet intensity only.

The ever-growing evidences indicate the importance of accounting for small-scale current structures for GIC estimates. The structure of the local magnetic field disturbance during these MIEs at single sites can differ greatly from regionally averaged fields (Pulkkinen et al., 2015). Time instances during which these extreme local peaks occur cover a wide range of local times (Ngwira et al., 2015). The events also appear at different latitudes, sometimes even at middle latitudes. The occurrence of such localized effect significantly deviates from the traditional storm time distribution pattern in which the regional 


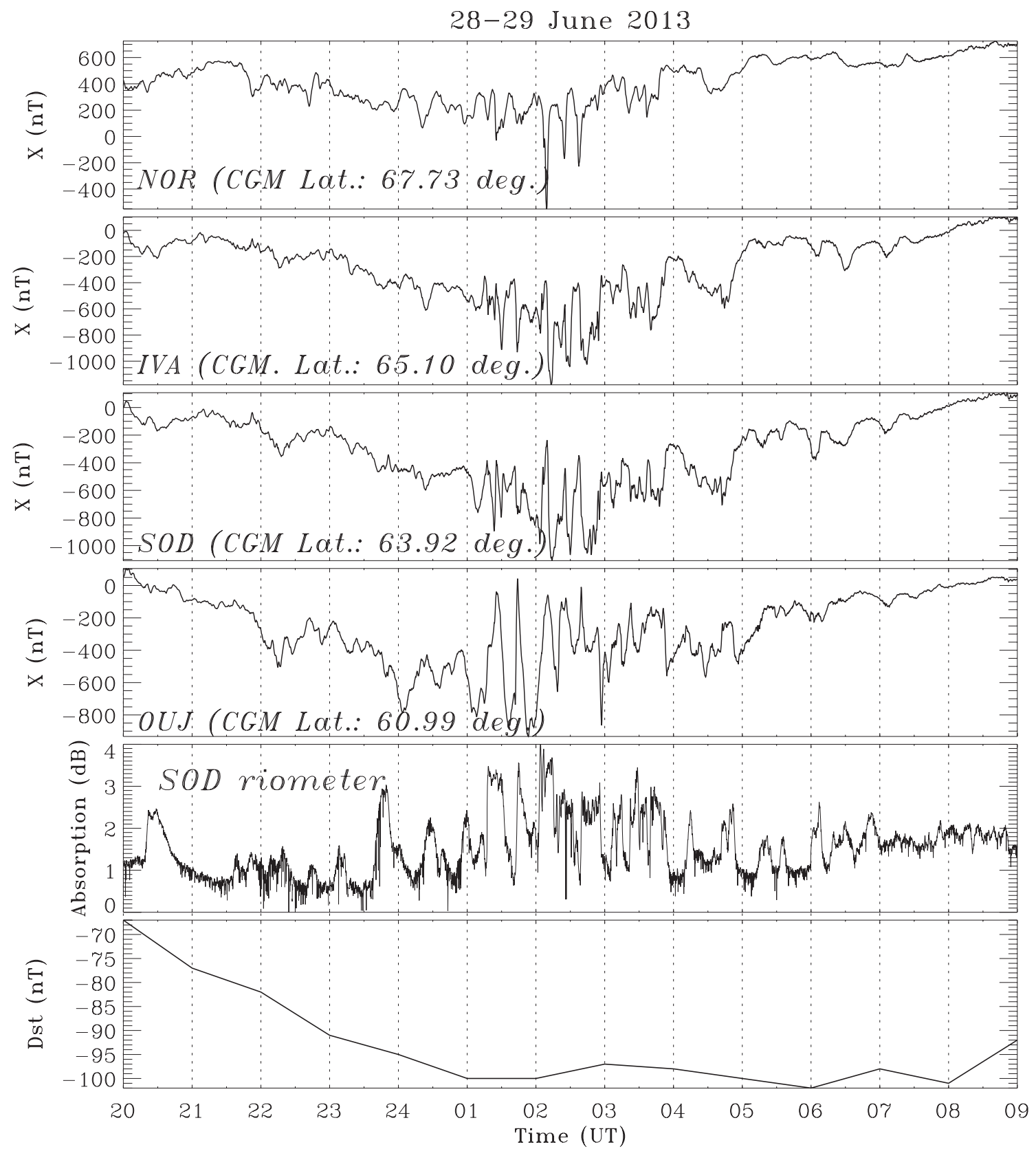

Fig. 11. $X$ component [nT] of geomagnetic fields at the latitudinal array of stations NOR, IVA, SOD, and OUJ (10-s data) during the magnetic storm between 20 UT on 28 June 2013 and 09 UT on 29 June 2013. The fifth panel shows the riometer absorption [relative units] at station SOD (10-s data). The bottom panel shows hourly Dst index [nT]. Geomagnetic latitudes are indicated near the station codes.

variations are assumed to be more or less uniform. The physical processes that govern the generation of these extremes have not been sufficiently explored.

The observed patterns of the $\mathrm{d} \boldsymbol{B} / \mathrm{d} t$ distribution demands an account of the magnetic field produced by non-stationary vortex-like structures, produced by localized field-aligned currents flowing in/out the ionosphere. The amplitudes of currents in such structures are not large and cannot greatly modify the storm-time $\boldsymbol{\Delta} \boldsymbol{B}$ distribution. However, their temporal variations are fast and can substantially influence the distribution of $\mathrm{d} \boldsymbol{B} / \mathrm{d} t$.
Local magnetic field variations $\mathrm{d} \boldsymbol{B} / \mathrm{d} t$ are caused by temporal fluctuations $\partial_{t} B(t)$ and movement of inhomogeneous magnetic structures along the ionosphere with velocity $\boldsymbol{V}$, namely $\mathrm{d} \boldsymbol{B} \mathrm{d} t=\partial_{t} \boldsymbol{B}+(\boldsymbol{V} \nabla) \boldsymbol{B}$. The electromagnetic structures associated with impulsive processes not only vary rapidly in time, but they may propagate along the ionosphere with high velocities, around $10-100 \mathrm{~km} \mathrm{~s}^{-1}$, and the latter factor may be significant for them. In particular, magnetic field variations produced by $\mathrm{Pi} 3$ pulsations are composed from time variations and variations caused by fast azimuthal drift of these structures. 


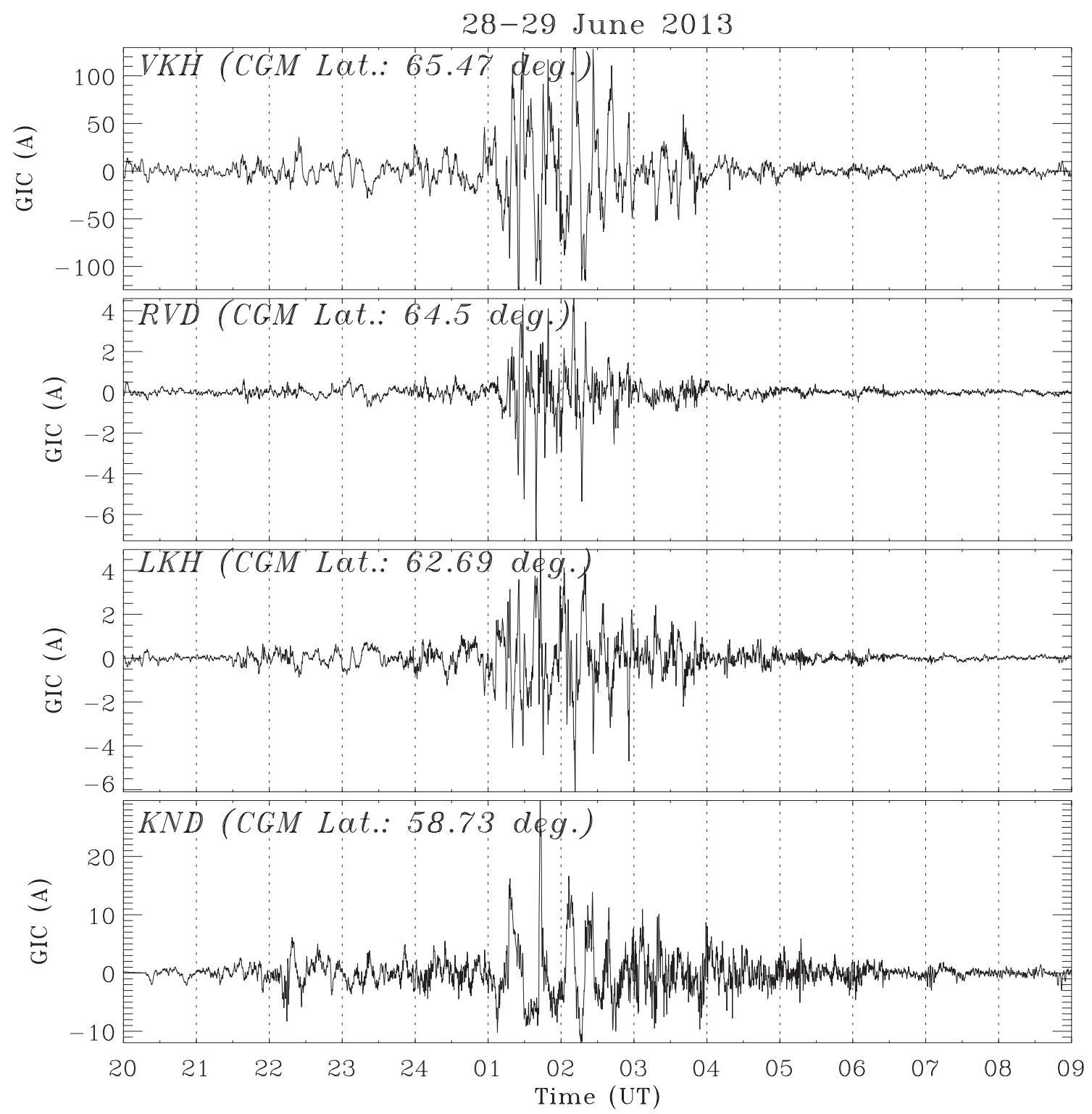

Fig. 12. GIC [A] at stations VKH, RVD, LKH, and KND (1-min data) between 20 UT on 28 June and 09 UT on 29 June, 2013. Corrected geomagnetic latitudes are shown near station codes.

The relationship between the large-scale auroral electrojet and localized current systems superposed on it during substorms has not been resolved. The necessity to take into account $2 \mathrm{D}$ features of the ionospheric current system for GIC modeling during severe disturbances was also demonstrated by Apatenkov et al. (2004). Using analytical approximations for a linear current and a vortex-like system, they decomposed a magnetic disturbance event with large $\mathrm{d} \boldsymbol{B} / \mathrm{d} t$ recorded by $2 \mathrm{D}$ magnetometer array into separate contributions from the E-W electrojet and the current vortex. On average, the number of events with dominant contribution from either electrojet or vortex were nearly the same, but the largest number of events with fast geomagnetic variations $\left(\mathrm{d} B / \mathrm{d} t>1.7 \mathrm{nT} \mathrm{s}^{-1}\right)$ were produced by vortex transient systems in the morning hours. Thus, the role of vortex current systems imposed on the auroral electrojet for GIC production increased with growth of $\mathrm{d} \boldsymbol{B} / \mathrm{d} t$. The spatial scale of vortex current systems could not be estimated because of the oversimplicity of the model used.

Thus, though the largest magnetic disturbances are produced by the auroral electrojet and oriented in the N-S direction, rapid variations of the geomagnetic field essential for GIC excitation are considerably determined by small-scale current systems, which disturb both horizontal components of the geomagnetic field. Evident confirmation of this fact is the noticeable vulnerability of Kola power lines extended in the N-S direction to GIC occurrence. There is no one-to-one relationship between the substorm intensity and the level of geomagnetic field variability. Sporadic intense night-time MIE could be the manifestation of intermittency of magnetic turbulence during substorms. They could be a result of transient processes in the magnetotail, such as super-strong bursty bulk flows. The physics 


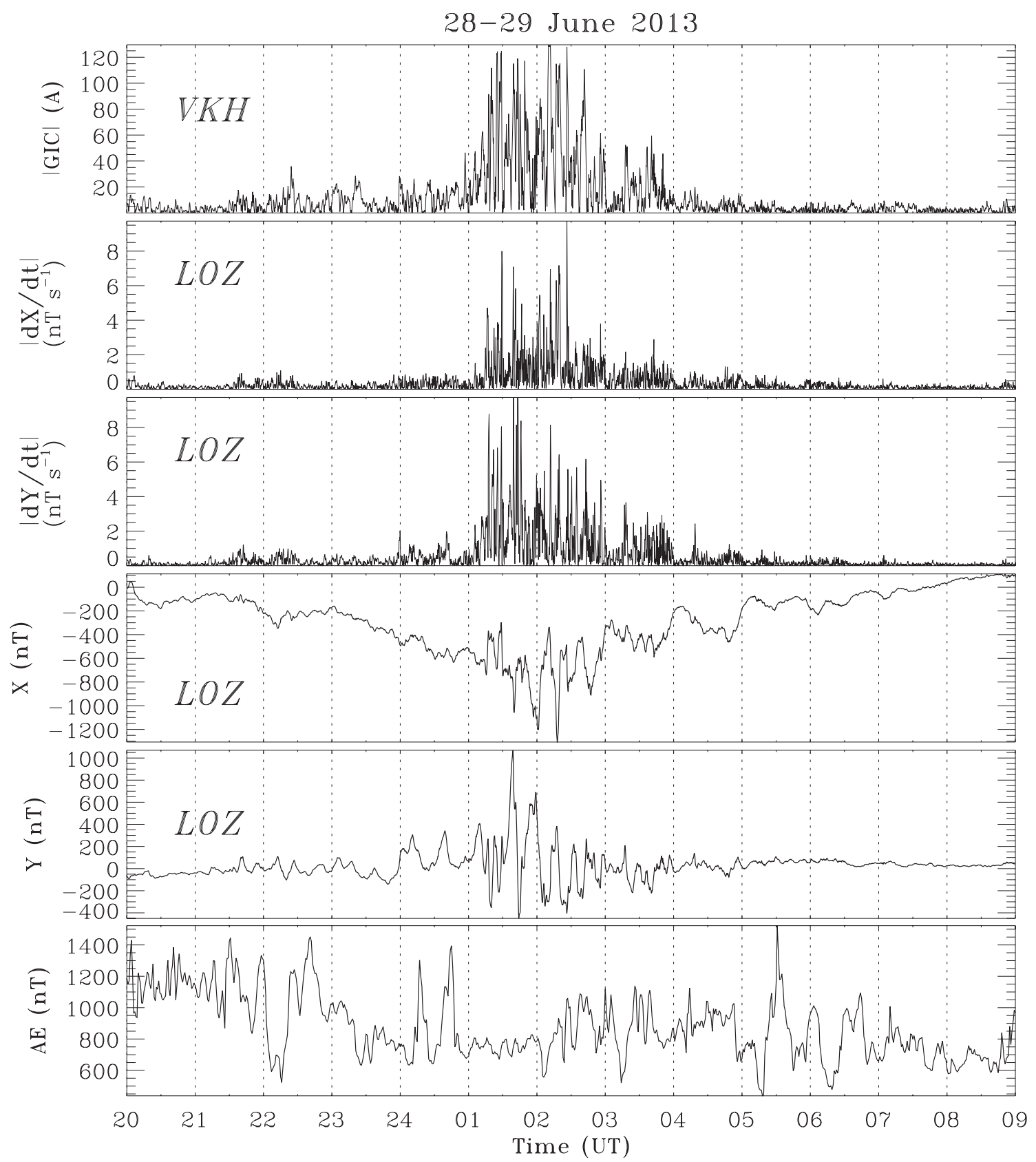

Fig. 13. Comparison between GIC amplitude [A] (10-s cadence), amplitudes of the time derivatives $|\mathrm{d} X / \mathrm{d} t|$ and $|\mathrm{d} Y / \mathrm{d} t|\left[\mathrm{nT} \mathrm{s}^{-1}\right]$, and $X$ and $Y$ components of geomagnetic field [nT] at LOZ (closely spaced to VKH) between 20 UT on 28 June 2013 and 09 UT on 29 June 2013 (10-s cadence). The bottom panel shows variations of the 1-min AE index.

of sporadic magnetic impulses during substorm evolution has not been established.

Calculation of geoelectric fields and GIC requires a sufficiently dense network of magnetometers and information about the geoelectric structure of the Earth. A comprehensive global model of geoelectric conductivity does not exist so far, therefore the calculations should use different approximation schemes. Comparison of different methods showed that with a good accuracy for calculating the telluric fields, one can use the impedance ratio in the approximation of a plane wave and plane geometry (Vilijanen et al., 2004). Greatly simplifying the situation is the fact that for the practically important assessment of GIC it is sufficient to provide integral estimates of the potential difference between the nodes of an extended system (the relevant length scale is the distance between grounded points of a power grid, and it varies typically from some $10 \mathrm{~km}$ to some $100 \mathrm{~km}$ ), and therefore necessary accuracy can be achieved with a relatively sparse network of magnetometers and a rough model of the crust's conductivity. However, this approximation may be questionable for modeling the GIC from localized structures, such as TCVs, substorm-associated pulses, and Pi3 pulsations. 


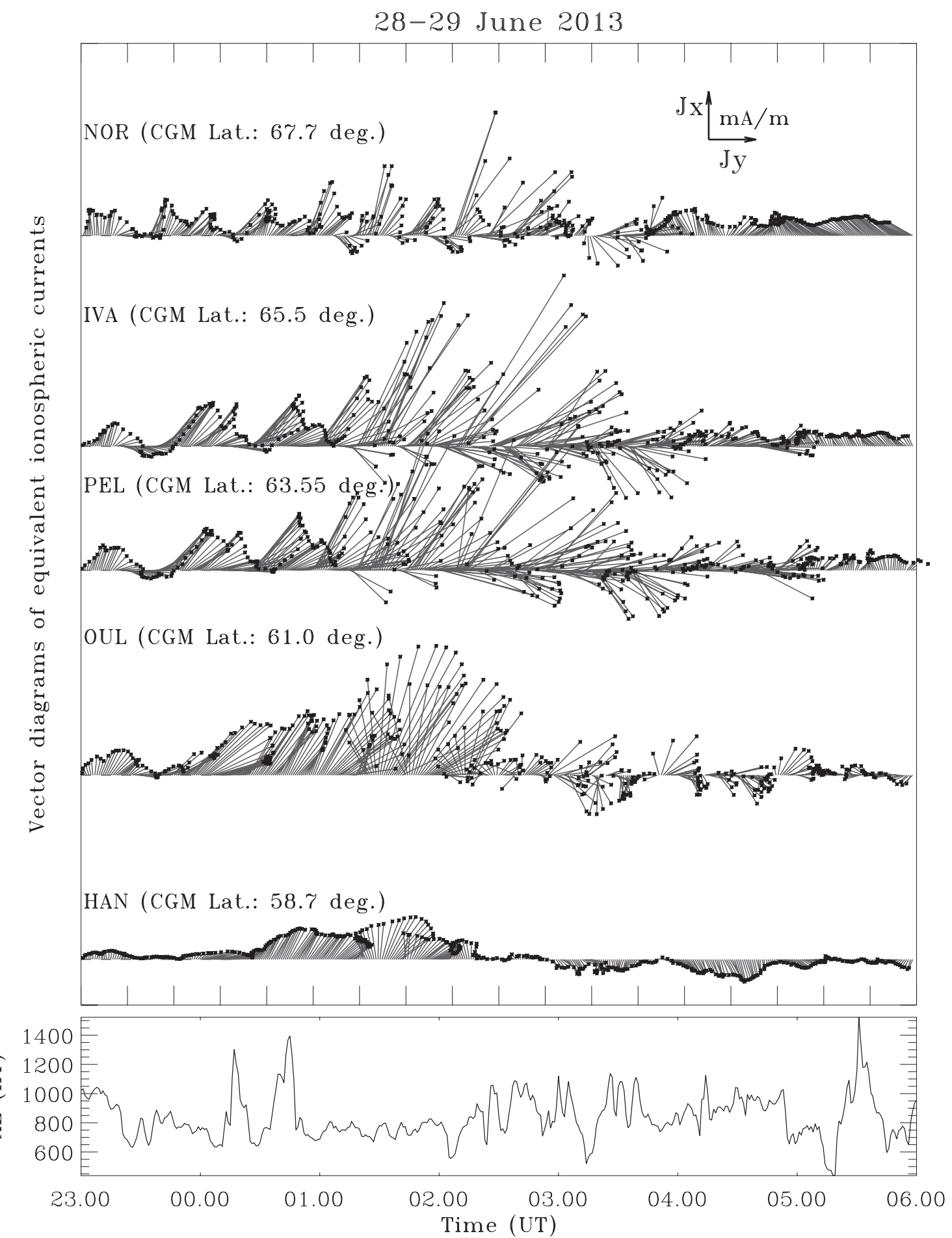

Fig. 14. Vector diagrams of 1-min equivalent ionospheric current $\boldsymbol{J}$ corresponding to Pi3 pulsations for the period from 23 UT on June 28 to 06 UT on June 29, with time cadence $1 \mathrm{~min}$.

\section{Conclusion}

Some types of the presented impulsive events (e.g., SC/SI) have been discussed already in regard to GIC excitation, but some types have not been seriously considered as a GIC driver (TCVs, Pi3 pulsations) to the best of our knowledge. At auroral latitudes, the large-scale structure of the $X$-component of the disturbed geomagnetic field is mainly determined by the ionospheric East-West electrojet. In smaller regional scales $\left(<10^{3} \mathrm{~km}\right)$, weaker but rapidly varying localized vortex-like current systems are superposed on the electrojet. The vector diagram technique has shown a much larger variability of the time 
derivative $\mathrm{d} \boldsymbol{B} / \mathrm{d} t$ both in magnitude and direction as compared with magnetic field disturbance $\Delta \boldsymbol{B}$. These localized current structures produce intense GICs, as observed by the GIC recording system of the power lines in the Kola Peninsula. The nightside solitary vortices observed as magnetic pulses with large amplitudes superposed on the substorm-related magnetic bay have been observed to be accompanied by very intense GICs (peak-to-peak variations about $70 \mathrm{~A}$ ). Such magnetic impulses embedded into a substorm are of interest well beyond the GIC community, because of their unresolved physical mechanism. A quasi-periodic sequence of localized vortex-like structures observed by magnetometers as $\mathrm{Pi} 3$ pulsations produces very high values of GIC (up to $\sim 120 \mathrm{~A}$ ).

Noticeable GIC can be generated on the day-side even during geomagnetically quiet conditions. Burst of GIC may be caused by global SC/SI pulses and TCVs. GIC pulses excited by $\mathrm{SC} / \mathrm{SI}$ can be even more intense than that generated during the substorm onset. The TCV magnetic disturbances are more localized, so intense GIC excitation is possible only if TCVs are generated in the vicinity of a power line.

These results have confirmed that GIC cannot be modeled by a simple model of the extended ionospheric current and dictate the necessity to take into account superposed localized vortex-like current systems. The identification and modeling of GIC drivers demands a detailed knowledge of fundamental substorm features, such as its fine temporal and spatial structure, and correspondence between quasi-static and impulsive components. There are many ongoing efforts to nowcast and predict a regional GIC intensity based on the magnetohydrodynamic (MHD) modeling of the geospace down to the surface of the Earth augmented by a coupled forward modeling of geoelectric field (e.g., Ivannikova et al., 2018). Because the existing MHD models are hardly able to reproduce small-scale nightside magnetic impulsive events, these efforts may be insufficient to predict realistic GIC bursts.

Acknowledgements. This study is supported by the grant no. 16-17-00121 from the Russian Science Foundation (SYA), the U.S. National Science Foundation grants AGS-1651263 to Augsburg University (EMJ), and PLR-1744828 to Virginia Tech (PVA). The GIC data are available at the website (http:// eurisgic.org). We thank the national institutes that support the IMAGE magnetic observatories (http://www.ava.fmi.fi/ image). The interplanetary parameters were taken from the OMNI database (https://omniweb.gsfc.nasa.gov). We appreciate thorough and in-depth examination of the manuscript by all Reviewers. The editor thanks Ari Viljanen and Shinichi Watari for their assistance in evaluating this paper.

\section{References}

Amm O, Viljanen A. 1999. Ionospheric disturbance magnetic field continuation from the ground to the ionosphere using spherical elementary current systems. Earth Planets Space 51: 431-440. DOI: $10.1186 / \mathrm{BF} 03352247$.

Anderson CW, Lanzerotti LJ, Maclennan CG. 1974. Outage of the L-4 system and the geomagnetic disturbances of August 4, 1972. Bell Syst Techn J 53: 1817.
Apatenkov SV, Sergeev VA, Pirjola R, Viljanen A. 2004. Evaluation of the geometry of ionospheric current systems related to rapid geomagnetic variations. Ann Geophys 22: 63-72.

Araki T. 1994. A physical model of the geomagnetic sudden commencement. In: Solar wind sources of magnetospheric ultralow-frequency waves, Engebretson MJ, Takahashi K, Scholer M (Eds.), AGU, Washington, D.C., pp. 183-200.

Belakhovsky VB, Pilipenko VA, Sakharov YaA, Lorentzen DL, Samsonov SN. 2017. Geomagnetic and ionospheric response to the interplanetary shock on January 24, 2012. Earth Planets Space 69(105): 2017. DOI: 10.1186/s40623-017-0696-1.

Belakhovsky VB, Pilipenko VA, Sakharov YaA, Selivanov VN. 2018. Characteristics of the geomagnetic field variability for the study of the magnetic storm and substorm impact on electrical power systems. Fizika Zemli (Solid Earth Phys) N1: 173-185.

Béland J, Small K. 2004. Space Weather Effects on Power Transmission Systems: The Cases of Hydro-Québec and Transpower New Zealand Ltd. In: Effects of space weather on technology infrastructure. NATO science series II: mathematics, physics and chemistry, Daglis IA (Ed.), vol. 176, Springer, Dordrecht.

Boteler DH, Pirjola RJ, Nevanlinna H. 1998. The effects of geomagnetic disturbances on electrical systems at the Earth's surface. $A d v$ Space Res 22: 17-27. DOI: 10.1016/S0273-1177(97)01096-X.

Carter BA, Yizengaw E, Pradipta R, Halford AJ, Norman R, Zhang K. 2015. Interplanetary shocks and the resulting geomagnetically induced currents at the equator. Geophys Res Lett 42: 6554-6559. DOI: 10.1002/2015GL065060.

Engebretson MJ, Yeoman TK, Oksavik K, Søraas F, Sigernes F, et al. 2013. Multi-instrument observations from Svalbard of a traveling convection vortex, electromagnetic ion cyclotron wave burst, and proton precipitation associated with a bow shock instability. $J$ Geophys Res 118: 2975-2997. DOI: 10.1002/jgra.50291.

Erinmez IA, Kappenman JG, Radasky WA. 2002. Management of the geomagnetically induced current risks on the national grid company's electric power transmission system. J Atmos Sol-Terr Phys 64: 743-756.

Eroshenko EA, Belov AV, Boteler D, Gaidash SP, Lobkov SL, Pirjola R, Trichtchenko L. 2010. Effects of strong geomagnetic storms on Northern railways in Russia. Adv Space Res 46: 1102-1110. DOI: 10.1016/j.asr.2010.05.017.

Fiori RAD, Boteler DH, Gillies DM. 2014. Assessment of GIC risk due to geomagnetic sudden commencements and identification of the current systems responsible. Space Weather 12: 76-91. DOI: 10.1002/2013SW000967.

Forbes KF, St. Cyr OC. 2004. Space weather and the electricity market: An initial assessment. Space Weather 2: S10003. DOI: 10.1029/2003SW000005.

Forbes KF, St. Cyr OC. 2008. Solar activity and economic fundamentals: Evidence from 12 geographically disparate power grids. Space Weather 6(10): s10003. DOI: 10.1029/2007SW000350.

Friis-Christensen E, McHenry MA, Clauer CR, Vennerstroem S. 1988. Ionospheric traveling convection vortices observed near the polar cleft: A triggered response to sudden changes in the solar wind. Geophys Res Lett 15: 253-256. DOI: 10.1029/GL015i003p00253.

Girgis R, Vedante K, Gramm K. 2012. Effects of geomagnetically induced currents on power transformers and power systems. CIGRE (Conseil International des Grands Réseaux Électriques) A2-304: 8.

Ivannikova E, Kruglyakov M, Kuvshinov A, Rastätter L, Pulkkinen A. 2018. Regional 3-D modeling of ground electromagnetic field due to realistic geomagnetic disturbances. Space Weather 16: 476-500. DOI: 10.1002/2017SW001793.

Juusola L, Kauristie K, van de Kamp M, Tanskanen EI, Mursula K, et al. 2015. Solar wind control of ionospheric equivalent currents 
and their time derivatives. J Geophys Res 120: 4971-4992. DOI:10.1002/2015JA021204.

Kappenman JG. 2003. Storm sudden commencement events and the associated geomagnetically induced current risks to ground-based systems at low-latitude and midlatitude locations. Space Weather 1: 1016. DOI: $10.1029 / 2003$ SW000009.

Kappenman JG. 2005. An overview of the impulsive geomagnetic field disturbances and power grid impacts associated with the violent sun-earth connection events of 29-31 October 2003 and a comparative evaluation with other contemporary storms. Space Weather 3: S08C01. DOI: 10.1029/2004SW000128

Kelly GS, Viljanen A, Beggan CD, Thomson AWP. 2017. Understanding GIC in the UK and French high-voltage transmission systems during severe magnetic storms. Space Weather 15: 99114. DOI: $10.1002 / 2016$ SW001469.

Kleimenova NG, Kozyreva OV, Kauristie K, Manninen J, Ranta A. 2002. Case studies on the dynamics of $\mathrm{Pi} 3$ geomagnetic and riometer pulsations during auroral activations. Ann Geophys 20: 151-159.

Knipp DJ. 2015. Synthesis of geomagnetically induced currents: Commentary and research. Space Weather 13: 727-729. DOI: 10.1002/2015SW001317.

Lanzerotti LJ. 2001. Space weather effects on technologies. Space Weather Geophys Monogr Ser AGU 125: 11.

Lanzerotti LJ, Wolfe A, Trivedy N, Maclennan CG, Medford LV. 1990. Magnetic impulse events at high latitudes: magnetopause and boundary layer plasma processes. J Geophys Res 95: 97-107. DOI: 10.1029/JA095iA01p00097.

Li H, Wang C, Xu WY, Kan JR. 2012. Characteristics of magnetospheric energetics during geomagnetic storms. J Geophys Res 117: A04225. DOI: 10.1029/2012JA017584.

Luo B, Li X, Temerin M, Liu S. 2013. Prediction of the AU, AL, and $\mathrm{AE}$ indices using solar wind parameters. J Geophys Res 118: 7683-7694. DOI: 10.1002/2013JA019188.

Marshall RA, Dalzell M, Waters CL, Goldthorpe P, Smith EA. 2012. Geomagnetically induced currents in the New Zealand power network. Space Weather 10: s08003. DOI: 10.1029/2012SW000806.

McHenry MA, Clauer CR. 1987. Modeled ground magnetic signatures of flux transfer events. J Geophys Res 92: 11231-11240. DOI:10.1029/JA092iA10p11231.

Nagano H, Suzuki A, Kim JS, Sugiura M. 1981. Pi3 magnetic pulsations associated with substorms. Planet Space Sci 29: 529-553. DOI: 10.1016/0032-0633(81)90067-2.

Newell PT, Gjerloev JW. 2011. Substorm and magnetosphere characteristic scales inferred from the SuperMAG auroral electrojet indices. J Geophys Res 116: A12232. DOI: 10.1029/2011JA016936.

Ngwira CM, Pulkkinen AA, Bernabeu E, Eichner J, Viljanen A, Crowley G. 2015. Characteristics of extreme geoelectric fields and their possible causes: Localized peak enhancements. Geophys Res Lett 42: 6916-6921. DOI: 10.1002/2015GL065061.

Pirjola R. 2000. Geomagnetically induced currents during magnetic storms. IEEE Trans Plasma Sci 28: 1867-1873. DOI: 10.1109/ 27.902215.

Pirjola R, Kauristie K, Lappalainen H, Viljanen A, Pulkkinen A. 2005. Space weather risk. Space Weather 3: S02A02. DOI: 10.1029/2004SW000112.

Pulkkinen A, Pirjola R, Boteler D, Viljanen A, Yegorov I. 2001. Modeling of space weather effects on pipelines. J Appl Geophys 48: 233-256.

Pulkkinen A, Amm O, Viljanen A, BEAR Working Group. 2003. Ionospheric equivalent current distributions determined with the method of spherical elementary current systems. J Geophys Res 108: 1053. DOI: 10.1029/2001JA005085.
Pulkkinen A, Lindahl S, Viljanen A, Pirjola R. 2005. Geomagnetic storm of 29-31 October 2003: Geomagnetically induced currents and their relation to problems in the Swedish high-voltage power transmission system. Space Weather 3(8): S08C03. DOI: 10.1029/ 2004SW000123.

Pulkkinen A, Klimas A, Vassiliadis D, Uritsky V, Tanskanen E. 2006. Spatiotemporal scaling properties of the ground geomagnetic field variations. J Geophys Res 111: A03305. DOI: 10.1029/ 2005JA011294.

Pulkkinen A, Bernabeu E, Eichner J, Viljanen A, Ngwira C. 2015. Regional-scale high-latitude extreme geoelectric fields pertaining to geomagnetically induced currents. Earth, Planets and Space 67: 93. DOI: $10.1186 / \mathrm{s} 40623-015-0255-6$.

Pulkkinen A, Bernabeu E, Thomson A, Viljanen A, Pirjola R, et al. 2017. Geomagnetically induced currents: Science, engineering and applications readiness. Space Weather 15: DOI: 10.1002/ 2016 SW001501.

Saito T. 1978. Long-period irregular magnetic pulsations Pi3. Space Sci Rev 21: 427-467. DOI: 10.1007/BF00173068.

Sakharov YaA, Danilin AN, Ostafiychuk RM. 2007. Registration of GIC in power systems of the Kola Peninsula. Proc. of 7-th symp. on Electromagnetic Compatibility and Electromagnetic Ecology, Institute of Electrical and Electronics Engineers (IEEE), St-Petersburg: 291-293.

Sakharov YaA, Kudryashova NV, Danilin AN, Ostafiychuk RM, Saranskiy SN. 2009a. Geomagnetic disturbances and railway automatic failures. Proc. of 8th Intern. Symp. on Electromagnetic Compatibility and Electromagnetic Ecology, Institute of Electrical and Electronics Engineers (IEEE), St-Petersburg: 235-236.

Sakharov YaA, Danilin AN, Ostafiychuk RM, Katkalov YuV, Kudryashova NV. 2009b. Geomagnetically induced currents in the power systems of the Kola peninsula at solar minimum. Proc. of 8th Symp. on Electromagnetic Compatibility and Electromagnetic Ecology, Institute of Electrical and Electronics Engineers (IEEE), St-Petersburg: 237-238.

Schrijver CJ, Dobbins R, Murtagh W, Petrinec SM. 2014. Assessing the impact of space weather on the electric power grid based on insurance claims for industrial electrical equipment. Space Weather 12: 487-498. DOI: 10.1002/2014SW001066.

Schulte in den Baumen H, Moran D, Lenzen M, Cairns I, Steenge A. 2014. How severe space weather can disrupt global supply chains. Nat Hazards Earth Syst Sci 14: 2749-2759. DOI: 10.5194/nhess14-2749-2014.

Trivedi NB, Vitorello A, Kabata W, Dutra SLG, Padilha AL, et al. 2007. Geomagnetically induced currents in an electric power transmission system at low latitudes in Brazil: A case study. Space Weather 5(4): 1-10: DOI: 10.1029/2006SW000282.

Vakhnina VV, Kuvshinov AA, Shapovalov VA, Chernenko AN, Kretov DA. 2015. The development of models for assessment of the geomagnetically induced currents impact on electric power grids during geomagnetic storms. Adv Elect Comp Eng 15: 49-54. DOI: 10.4316/AECE.2015.01007.

Viljanen A. 1997. The relation between geomagnetic variations and their time derivatives and implications for estimation of induction risks. Geophys Res Lett 24: 631-634. DOI: 10.1029/97GL00538.

Viljanen A. 1998. Relation of geomagnetically induced currents and local geomagnetic field variations. IEEE Trans Power Delivery 13: 1285-1290. DOI: 10.1109/61.714497.

Viljanen A. 2011. European project to improve models of geomagnetically induced currents. Space Weather 9: S07007. DOI: 10.1029/2011SW000680. 
Viljanen A, Nevanlinna H, Pajunpaa K, Pulkkinen A. 2001. Time derivative of the geomagnetic field as an activity indicator. Ann Geophys 19: 1107-1118.

Viljanen A, Pulkkinen A, Amm O, Pirjola R, Korja T, BEAR Working Group. 2004. Working Group. Fast computation of the geoelectric field using the method of elementary current systems and planar Earth models. Ann Geophys 22: 101-113.

Viljanen A, Wintoft P, Wik M. 2015. Regional estimation of geomagnetically induced currents based on the local magnetic or electric field. J Space Weather Space Clim 5: A24. DOI: 10.1051/ swsc/2015022.

Vorobjev VG, Yagodkina OI, Zverev VL. 1999. Morphological features of bipolar magnetic impulsive events and associated interplanetary medium signatures. J Geophys Res 104: 4595-4608.

Watari S, Kunitake M, Kitamura K, Hori T, Kikuchi T, et al. 2009. Measurements of geomagnetically induced current in a power grid in Hokkaido, Japan. Space Weather 7(3): s03002. DOI: 10.1029/ 2008 SW000417.
Weigel RS, Klimas AJ, Vassiliadis D. 2003. Solar wind coupling to and predictability of ground magnetic fields and their time derivatives. J Geophys Res 108: 1298. DOI: 10.1029/ 2002JA009627.

Wintoft P, Wik M, Viljanen A. 2015. Solar wind driven empirical forecast models of the time derivative of the ground magnetic field. J Space Weather Space Clim 5: A7. DOI: 10.1051/swsc/ 2015008.

Zanetti L, Anderson B, Potemra T, Kappenman J, Lesher R, Feero W. 1994. Ionospheric currents correlated with geomagnetic induced currents: Freja magnetic field measurements and the Sunburst Monitor System. Geophys Res Lett 21: 1867-1870. DOI: 10.1029/94GL01425.

Zhang JJ, Wang C, Sun TR, Liu CM, Wang KR. 2015. GIC due to storm sudden commencement in low-latitude high-voltage power network in China: Observation and simulation. Space Weather 13: 643-655. DOI: 10.1002/2015SW001263.

Cite this article as: Belakhovsky V, Pilipenko V, Engebretson M, Sakharov Y \& Selivanov V. 2019. Impulsive disturbances of the geomagnetic field as a cause of induced currents of electric power lines. J. Space Weather Space Clim. 9, A18. 